\title{
Les autels portatifs et leurs inscriptions
}

Robert Favreau

\section{Citer ce document / Cite this document :}

Favreau Robert. Les autels portatifs et leurs inscriptions. In: Cahiers de civilisation médiévale, 46e année ( $\left.{ }^{\circ} 184\right)$, Octobredécembre 2003. pp. 327-352;

doi : https://doi.org/10.3406/ccmed.2003.2865

https://www.persee.fr/doc/ccmed_0007-9731_2003_num_46_184_2865

Fichier pdf généré le 25/03/2019 


\title{
Résumé
}

Les plus anciennes inscriptions des autels portatifs portent les noms des donateurs, l'état des reliques, le rappel des consécrations. Au Xle s. les inscriptions commémorent aussi la croix glorieuse, louent le Dieu très saint (Bamberg, Berlin, Brunswick), rappellent la vie du Christ (Namur), citent vertus cardinales, fleuves du Paradis (Munich, Fritzlar, Bruxelles). Le Xlle siècle est marqué d'une part par le retour de l'Ancien Testament, prophètes et apôtres, Église et Synagogue (autels d'Eilbert de Cologne, de Saint-Maurice de Siegburg, de Stavelot), d'autre part par l'insistance sur la Présence réelle dans l'eucharistie, suite à la controverse bérengarienne (autels de Saint-Grégoire à Siegburg, de Bamberg, Cologne, Berlin, Paderbon, Londres, Oloron-Sainte-Marie, Liège).

\begin{abstract}
The oldest inscriptions of the portable altars bear the name of donators, the state of relies and remind the consecrations. During the XIth century, the inscriptions commemorate also the Glorious Cross, the very Holy God (Bamberg, Berlin, Brunswick), remind the Life of Christ (Namur), quote Cardinal Virtues, the Rivers of Paradise (Munich, Fritzlar, Bruxelles). The XIlth Century is marked on the one hand by the return of the Old Testament, prophets and apostles, Church and Synagoga (altars of Eilbert of Cologna, St. Maurice of Siegburg, of Stavelot), and on another hand by the insistence of the Real Presence in the eucharist, after the theological controverse of Berenger of Tours (altars of St. Gregory at Siegburg, Bamberg, Cologna, Berlin, London, Oloron-Sainte-Marie, Liège).
\end{abstract}




\title{
Robert FAVREAU
}

\section{Les autels portatifs et leurs inscriptions}

\begin{abstract}
RISTMI:
Les plus anciennes inscriptions des autels portatifs portent les noms des donateurs. l'état des reliques. le rappel des consécrations. Au XIE s. les inscriptions commémorent aussi la croix glorieuse. louent te Dieu très saint (Bamberg. Berlin. Brunswick), rappellent la vie du Christ (Namur). citent vertus cardinales. fleuves du Paradis (Munich. Fritzlar. Bruxelles). Le XII" siècle est marqué d'une part par le retour de l'Ancien Testament. prophètes et apôtres. Église et Synagogue (autels d'Filbert de Cologne. de Saint-Maurice de Siegburg. de Stavelot). dautre part par l'insistance sur la Présence réelle dans leucharistie, suite à la controverse bérengarienne (autels de Saint-(irégoire à Siegburg. de Bamberg. Cologne. Berlin. Paderbon. Londres. Oloron-Sainte-Maric. Liège).
\end{abstract}

\section{ABSTRAC"I}

The oldest inscriptions of the portable altars bear the name of donators. the state of relies and remind the consecrations. During the XIth century. the inscriptions commemorate also the Glorious (ross. the very Holy God (Bamberg. Berlin. Brunswick). remind the Life of Christ (Namur). quote Cardinal Virtues, the Rivers of Paradise (Munich. Fritzlar. Bruxelles). The XIIth Century is marked on the one hand by the return of the Old Testament. prophets and apostles. Church and Synagoga (altars of Eilbert of Cologna. St. Maurice of Siegburg. of Stavelot). and on another hand by the insistence of the Real Presence in the eucharist. after the theological controverse of Berenger of Tours (altars of St. Gregory at Sieghurg. Bamberg. Cologna. Berlin. London. Oloron-Sainte-Marie. Liege).

Il est probable que les autels portatifs aient été employés dès les premiers siècles '. mais leur histoire n’est vraiment assurée qu à partir du vil' $\mathrm{s}$. Le plus ancien qui nous soit parvenu est celui de saint Cuthbert $(\doteqdot$ 687). trouvé avec les ossements du saint dans la cathédrale de Durham et conservé aujourd hui dans la bibliothèque du chapitre. tablette de bois recouverte d'une feuille d’argent. avec l'inscription : IN HONOR... S. PETRU. Au dire de Siméon de Durham un autel portatif avait été trouvé sur la poitrine de saint Acca. évêque d’Hexham (740) : il était en bois avec clous d'argent et portait l'inscription ALMAE TRINITATI AGIE SOPHIE SANCTAE MARIAE?

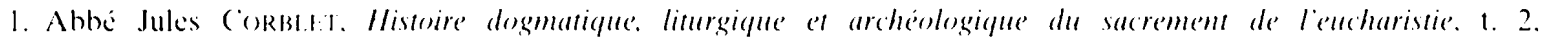
Paris Bruxellesi(icneve. 1886. p. 209.

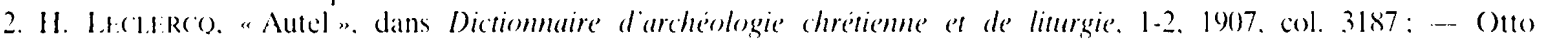

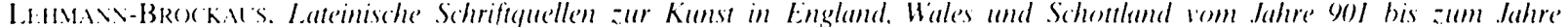
1.307. I. Munich. 1955. n 2096. p. 558: - Joseph BR.1 X. S.J. Der christliche Altur in seiner geschichlichen Entwicklumg. I. Munich. 1924. p. $+22-423$.

Cahiers de civilisation médiévale, 46. 20103, p. 327-352. 
Fin VII"-début VIII" s. saint Wulfran. évêque de Sens. célébrait la messe sur un autel portatif pendant une traversée en mer. et au vill s. Bède le Vénérable parle de deux missionnaires de Frise qui avaient un autel avec eux ${ }^{3}$.

À lépoque carolingienne sont affirmées, pour les autels portatifs. les deux conditions essentielles que la liturgie exigeait pour les autels fixes : la consécration par lévêque, et l'emploi de la pierre ${ }^{4}$. Plutôt qu une pierre simple. on emploiera une belle matière. marbre ${ }^{5}$. porphyre. jaspe. albâtre. onyx. serpentine. saphir, ivoire. Le concile d'Épone de 517 avait interdit, en son canon 26. de consacrer des autels qui ne seraient pas de pierre". La raison en est symbolique bien plutôt que biblique. car l'Ancien Testament cite aussi bien l'or et l'airain que la pierre pour un autel $^{7}$. C"est le Christ qui est signifié par l'autel ${ }^{8}$. Or le Seigneur s'est présenté comme la pierre rejetce par les bâtisseurs et devenue pierre d'angle". Paul rappelle aux Corinthiens "que les Hébreux. dans le désert sous la conduite de Moïse. ont tous mangé le même aliment spirituel (la manne), et tous ont bu le même breuvage spirituel (l'eau du rocher)... et le rocher était le Christ»- petra autem erat Christus (1 Cor X. 3-4). Rappelant cette identification paulinienne. Thomas d'Aquin indique que la pierre était le Christ parce que le corps du Christ a été enseveli dans une sépulture de pierre. et que de plus la pierre est solide et peut être trouvée partout ${ }^{10}$. Pour Guillaume Durand l'autel doit être de pierre. non à cause de la dureté. mais de la solidité de la foi, selon ce que le Seigneur a dit à Pierre : "Tu es pierre et sur cette pierre [c'est-à-dire sur cette fermeté inébranlable de la foi] je bâtirai mon Église " ". Pour ces mêmes raisons symboliques la pierre doit être d'un seul tenant ${ }^{12}$. L'obligation de consécration par l'évêque. rappelée à l'époque carolingienne. se trouve répétée che\% tous les auteurs postéricurs. et une rubrique existe dans le pontifical pour la consécration d'un autel portatif ${ }^{13}$. Dès le IVe $s$. s'était imposé l'usage de déposer des reliques dans les autels. Cette tradition sera aussi observée pour les autels portatifs, même si Guillaume Durand. qui la rappelle. semble dire quil peut arriver qu'on ne dispose pas de reliques des saints pour un autel portatif ${ }^{14}$. À partir du XIII' $s$. l'autorisation davoir un autel portatif devra être demandée au pape ${ }^{15}$.

Un autel portatif est fait pour la célébration de la messe et cette fonction doit être présente à l'esprit quand on examine son iconographie et ses inscriptions. En effet la pierre d'autel ne comporte presque jamais une iconographie ${ }^{1 t}$. Elle doit être assez grande, déclare Gérard de Cambrai dans sa Cemma ecclesiastica pour qu'on puisse y placer la patène et le pied du calice ${ }^{17}$.

3. J. CORBII:T (op). cit. n. 1). p. $2(0)$.

4. Capitulaire de (harlemagne de 769 ) en voyage... le prêtre ne peut célébrer la messe que sur une pierre consacrée par lévéque: Hincmar. archevếque de Reims : si la nécessité lexige... on devra emplover une table de marbre. d'ardoise ou d'autre pierre trés convenable qui aura été consacrée (par lévếque) (P/. 125. col. 794).

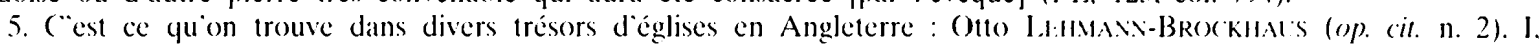
p. 331. n 1232. : duo altaria marmorea portabilia benedicta (Deverel). p. 572-574. n 21.57 : altare portabile marmoream benedictum (Horningshem) : II. p. 266 (Mere): III. p. 245 (Beccense monasterium).

6. Concile d’Épone. 517, canon 26: Altaria nisi lapidea chrismatis unctione non sacrentur (Sacrosancta concilia. éd. Ph. LABBi: et Gabriel (ossARI. IV. Paris. 1671. col. 1579: Les canoms des conciles merovingiens (Vle-VIle s.). éd. trad.

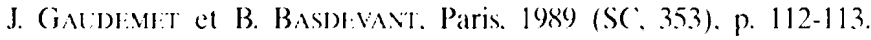

7. Ex XX. 25: XI. 5: Jos VIII. 31:1 R XVIII. 32:2 R XVI, 14 e 15:1 Mc I, 23: Ap VIII. 3. Référence peut aussi être faite à Jacob (C in XXVIII. 18) : Surgens ergo Jacob mane, tulit lapidem quem supposuerat capiti suo, et erexit in titulum. fiundens oleum ciesuper. citation qui est reprise au XIVe s. sur un autel portatif a Klagenfurt : + MANE SLRGENS JACOB ERIGEBAT ' I.APIDFM + IN TITULUM FUNDENS OIEUM DESLPER VOTUM (J. BRALN. op. (it. n. 2. I. p. 457).

8. Thomas D) Aot:N. Somme théologique. 3" pars. quaestio 83. articulus. 3.

9. Mt 21. 42 : 1.c 20.17: Ac 4. 11.

10. Thomas b Aotid. Somme théologique. 34. 4. 83. a. 3.

11. Rationale divinorum officiortum. 1. 1. c. 7. $\$ 25$. (suillaumme Durand cite ici Mt 16. 18.

12. (f. Ex 20. 25 : Quod si altare lapideum feceris mihi. non aedificabis illud de sectis lapidihus.

13. Pontifical de Ciuilloume Durand. éd. Michel Axis)RI:1. (ité du Vatican. 1941 (Studi è Iesti. 88). p. 498-504.

14. Rationale divinorum officiorum. I. 1. c. 7. \$23.

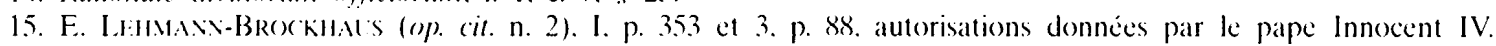

16. La croix nue tracée sur ke cristal de l'autel portatif dehenri II a la chambre du trésor de la résidence de Munich constitue une exception.

17. Distinctio 1. cap. 10. De ornamentis altaris, cité par (). Li:MMAN-BRo(KAes (op). cit. n. 2). III. p. 80). n 54.35 . Relevé de dimensions dans Jospeh BRAl' (op) (it. n. 2). I. p. 4.34-4.35. 
Hildebert de Lavardin précise même que le calice doit être placé à droite parce que le sang a coulé. sur la croix. du côté du Christ ${ }^{18}$. Patène et calice occulteraient une iconographie tracée sur la pierre dautel. et ils ont de plus leur propre iconographie. Le centre de la patène est luimême le plus souvent sans iconographic. car on y place la grande hostic sur laquelle le moule à hostie a imprimé Crucifixion. Agneau de Dieu ou une autre figure. C"est ainsi que pour ma part. je ne retiendrai pas la désignation d'autel portatif pour une plaque de cuivre doré et émaux champlevés du musée du Louvre du milieu du XIl' s. qui comporte. sur toute la plaque le dessin de Jésus dont on cloue pieds et mains sur la croix ${ }^{19}$. car elle ne comporte pas de pierre d’autel. ni d’emplacement réservé à la patène et au calice. Linscription qui court autour de la plaque, VINEA MEA ELECTA QUOMODO CONVERSA IN AMARITUDINEM ME CRUCIFICIS. "Ma vigne choisie. tu me crucifies comme si j"étais tournée en amertume". est inspirée du prophète Jérémic (II. 21). mais tirée de l'Office des ténèbres du vendredi saint ${ }^{20}$. Iconographie et épigraphie des autels portatifs se rapportent bien plutôt au jeudi saint quau vendredi saint, et lorsqu ils mettent en scène la Crucifixion. c'est ordinairement d'une façon plus large que la seule Passion. mais avec le complément de la Résurrection et de l'Ascension. de l'Agneau de l'Apocalypse. des apôtres et de l'Église qui poursuivent l'œuvre du Christ mort et ressuscité ${ }^{21}$. De même j'écarte de la liste des autels portatifs. pour les mêmes raisons. la plaque d’émail du musée de Vienne où le Christ est représenté en Majesté dans une mandorle entre les symboles des évangélistes, avec l'inscription : IN ME PRETERITUM PRESENS MANET ATQUE FUTURUM. "En moi demeurent passé. présent et futur " ${ }^{22}$. qui n’est pas en rapport direct avec une célébration eucharistique. $\mathrm{Si}$ ces deux plaques appartenaient à un autel portatif. ce ne pourrait être que des plaques inférieures.

On connaît mal les autels portatifs et leurs inscriptions avant la fin du $x^{c}$ s. La pierre dautel de l'église des Saintes-Maries-de-la-Mer, du IX" s. porte seulement + ALTARE SANCTI SALVATORIS ${ }^{23}$. celle de l'église Saint-Pierre-et-Saint-Rufin de Moutiers-sous-Argenton, du x $x^{c}$ s., HEC EST MAGNA SANCTI RUFINI CONFESSORIS ${ }^{24}$. Sur l'autel portatif consacré par l'évêque Roger de Champagne (996-1023), longtemps conservé au trésor de la cathédrale de Beauvais on lisait : PRIMUM CRUCI PRESUL LOCUM SANXI MARIAE POSTERUM ROTGERUS TERCIUM PETRO QUARTUM DEDI LUCIANO, "Roger, évêque, jai consacré ce lieu d'abord à la Croix. ensuite à Maric. troisièmement à Pierre: en quatrième je l'ai donné à Lucien ${ }^{25}$. Lautel portatif de San Pedro de Roda. au musée diocésain de Gérone. porte sur sa face supéricure une inscription déjà élaborée :

[H]IC VIRTUS TONANTIS EXAUDIT PIE ORANTEM

MERITA SANCTORUM POSSUNT ADJUVARI ORANTEM

"Ici la puissance de Celui qui tonne exauce qui prie avec ferveur.

Les mérites des saints peuvent aider celui qui prie».

18. PL. 171, col. 1180

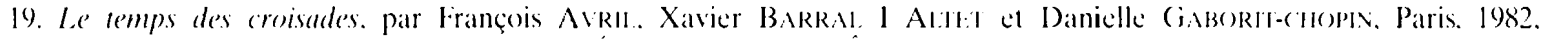

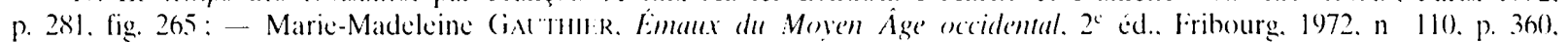
parle avec raison de "cassetle reliquaire dite probablement i tort. autel portatif" Jospeh BRAL:N (op. (it. 1). 2). I. p. 472. rejette aussi lappellation d'“autel portatif".

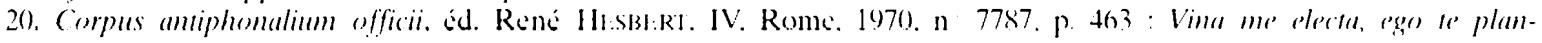
tari: quomodo comeres a in amaritudine ut me crucifigeres et Barraham dimitteres?"

21. Sur la cassette reliquaire du Lourre il y a hien les apotres sur lés cotés mais cela niest pas suffisant pour retenir lappellation dautel portatif.

22. ()esterreichisches Museum für angewandte Kunst. Inv.-NR. Fm. 399. ('T. Romanische Kumst in Oesterreich. Krems

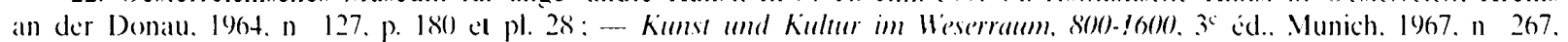
p. 586 et pl. 223 el 22ta. I.inscription est une transcription d $\lambda$ p IV. 8 : qui crat et qui est et qui renturus est.

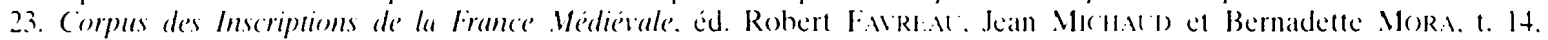
Paris. 1989. p. 138. lig. 99. labrige (I.F.M.).

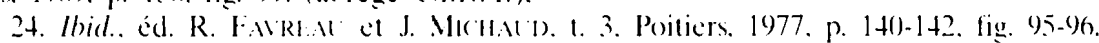

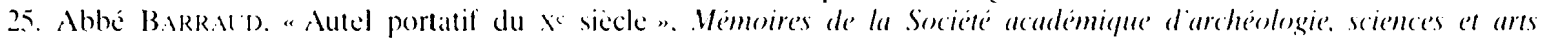

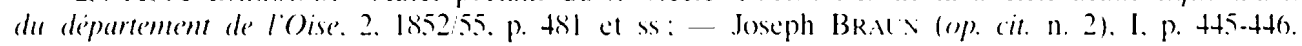


Il y a une certaine recherche de rime. mais en même temps l'écriture est fort grossière. Sur la face inférieure JHNS EGLT, Johannes evangelista. est représenté au milieu de la plaque tandis quau bord des longs côtés sont marqués les noms des donateurs. JOSUE ET ELIMBURGIS /FIERI JUSSERUNT ${ }^{26}$. On a déjà des autels portatifs d'une riche orfèvrerie. comme devait l'être lautel d’onyx décoré de lames d'or et d’argent que Geoffroi, archidiacre de Milan. envoyait à la fin du $x^{c}$ s. à Saint-Bénigne de Dijon et comme le montre lautel-reliquaire de Saint-André consacré par l'archevêque de Trèves Egbert (977-993) et conservé à la cathédrale. Autour de la pierre d'autel placée en l'espace restreint laissé par la sandale de saint André on peut lire : HOC ALTARE CONSECRATUM EST IN HONORE SANCTI ANDREAE APOSTOLI. et sur les bords de la plaque supérieure une longue inscription énumère les reliques déposées en lautel : HOC SACRUM RELIQUIARUM CONDITORIUM EGBERTUS ARCHIEPISCOPUS FIERI JUSSIT ET IN EO PIGNORA SANCTA SERVARI CONSTITUIT CLAVUM VIDELICET DOMINI ET DE SANCTI PETRI DE BARBA IPSIUS ET DE CATENA SANDALIUM SANCTI ANDREAE APOSTOLI ALIASQUE SANCTORUM RELIQUIAS QUAE SI QUIS AB HAC AECCLESIA ABSTULERIT ANATHEMA SIT ${ }^{27}$. Mais la grande période des autels portatifs se place au XI' et surtout au XII" s. avec une part prépondérante pour l'empire germanique.

Les noms des donateurs étaient. à Gérone. inscrits sur la plaque inférieure. Le plus souvent le donateur ou commanditaire a cependant préféré faire placer son nom sur le plaque supérieure. afin que le célébrant soit immédiatement incité à faire mémoire de lui au cours de la messe. Cétait le cas de l'évêque Roger à Beauvais. Au Musée de Berlin une pierre d’autel portatif est donnée par Adelvoldus. premier prévôt connu par les textes, pour l'église abbatiale Saint-Blaise de Brunswick (avant 1068-1100) : $†$ HUNC LAPIDEM CONSECRATUM DEDIT ADEVOLDUS PREPOSITUS SANCTO BLASIO ${ }^{2 \times}$. De même date un autel du trésor des Guelfes conservé aujourd hui au musée de Cleveland est un don de la comtesse Gertrude $(\dagger 1077)$ dont le nom est inscrit autour de la pierre d'autel :

(GERDRUDIS CHRISTO FELIX UT VIVAT IN IPSO

OBTULIT HUNC LAPIDEM (jEMMIS AUROQUE NITENTEM

"Afin qu'elle vive heurcuse dans le Christ. Gertrude

lui a offert cette pierre brillante de gemmes et d'or " ${ }^{29}$.

Linscription forme deux hexamètres léonins. un vers qui va devenir de règle dans la majorité des inscriptions accompagnant des cuvres de qualité. Une autre noble femme Svonehild offre avec dévotion un autel portatif à l'abbatiale de Melk dans la seconde moitié du XI" s., HOC ALTARE DEO SVONEHILD DEVOTA BENIGNO - également vers léonin ${ }^{30}$. Henri de Werl. évêque de Paderborn de 1090) à 1127. s'est fait représenter en bas de la pierre d’autel de la plaque supéricure de l'autel portatif qu'il a fait faire en 1100 par Roger d'Helmarshausen ${ }^{31}$ avec une oraison tirée du Psaume 141 : "Que ma prière devant toi s'élève comme un encens ». qui

26. Catalumya románicti. XX11I. Barcelone, 1988. p. 98-100).

27. Alfred DARCi:., "Trésor de Conques. Les autels portatifs". Amules archéologiques. 16. 1856. p. 79.

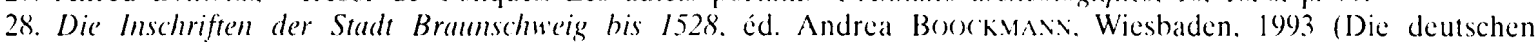
Inschriften, 35). n 7, p. 11 et fig. 9.

29. Ibidl. n 3. p. 5-7: - Hans Swar7t:Nsk1. Monuments of Romanesque Arts. The Art of Church Treasures in NorthWestern Europe. Londres. s. d.. p. 44. fig. 85. pl. 36:- Patrick M. D1. WISTr. R. The Sacral Treasure of the Cuephs, Cleveland. 1985. p. 37-40. pl. VI-IX: - Peter I.ASko. Ars sacra 800-1200\%. New-Haven/Londres. 1994. p. 136.

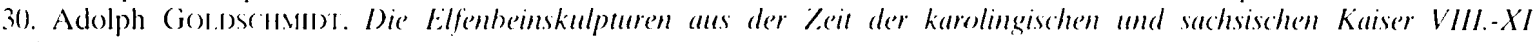
falurundert. 11. Burlin. 1970. p. 41). n 104. pl. XXXIII.

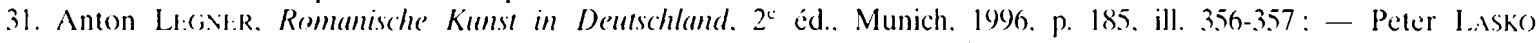
(op). (it n. 29). p. 16.3-164: . Patrick .1. D) Worte (op). (it. n. 29). p. 50. fig. 55. 
fait partie d'une prière de l'office du lundi ${ }^{32}$. Quatre vers léonins riches sur la plaque inférieure sont aussi consacrés à l'offrande de l'évêque. Dans le Sud-Ouest de la France. à la cathédrale d'Oloron-Sainte-Maric. une inscription rappelait. sur la table de l'autel portatif. les noms de l'auteur. Rainaud de Morlaas. et du commanditaire. l'évêque Roger de Saintes (110()-1114) ${ }^{3.3}$. Autour de la plaque de porphyre vert de l'autel de l'abbave de Cellanova aujourd hui au musée diocésain d'Orense en Galice. labbé Pierre (1090)-1118) a indiqué que c’était lui qui avait ordonné de faire cette oeuvre ${ }^{34}$. À la lin du Xlle $\mathrm{s}$. Wolbero donne un autel portatif ${ }^{35}$ et prie le Seigneur de lui être miséricordieux. en trois hexamètres léonins riches pleins d'humilité gravés autour de la plaque supéricure :

\title{
SIT DATOR AC DATUM TIBI (HRISTE PIISSIME (GRATUM \\ (IALDERE: (LALSTRA POLI DLM PULSAT WOLBERO NOLI \\ QLI TIBI DIEVOTLSS DIVINA CLEMENTIA MOTUS
}

"Que le donateur et le don te soient agréables. ô Christ dans ta grande bienveillance.

Veuille ne pas fermer les portes du ciel à Wolbero alors quil frappe

lui qui test tout dévoué et a confiance en la miséricorde divine".

À celte même époque l'impératrice Béatrice († 1184), seconde épouse de l'empereur Frédéric I ${ }^{\mathrm{E}}$. offre un autel portatif à l'archidiacre de Spire. "orné de gemmes el d'or» comme celui de la comtesse (iertrude un siècle plus tôt. et menace de lanathème quiconque l'en enlèverait ${ }^{3 / \text { ". Une }}$ semblable formule de condamnation figure dans l'inscription d'un autel portatif à Gandersheim ${ }^{37}$. On a encore le nom d'un donateur. THIDERICUS ABBAS III DEDIT, sur un autel portatif conservé au Bristish Museum: l'abbé a été identifié avec l'abbé de Scheiden près de Cologne. cité en $1226^{3{ }^{3 *}}$. Lexemple le plus remarquable d'une inscription de commanditaire est sans doute offert par les quatre vers léonins qui courent autour du cristal du riche autel portatif de la Sainte Croix conservé à la Schatzkammer der Residenz à Munich ${ }^{39}$ :

\author{
$\div$ EN (ESAR SOPHIAE RENITENS HEINRICUS HONORE \\ + CHRISTE ( REATORI DABIT HOC 'TIBI MUNUS HONORI \\ † IN OUO) SANCTA (RUCIS PARS CLAUDITUR AC DECUS ()RBIS \\ + REDDE VICLM PATRIAE DONANDO GAUDIA VERE
}

32. PS (XI.I (CXI). 2 : DIRICAATLR ORATIO) MEA SICUT INCENSLM IN (ONSPE("TO TL:O) DOMINI: DEUS. Le Psaume najoute pas Domine Deus. mais on notera qüil se poursuit par eleratio manum mearum sacrificium respertimum qui illustre lattitude du donateur. (ette priere lait partie de l'office du lundi (corpus amiphonalium officii.

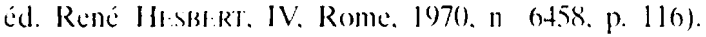

3.. (.I.K... 1. 6. Paris 1981. p. 162 :

HANC MORI.ANISNSIS RAINALDIS (ONDIDIT ARAM

PRAFSII. ROCIIRIOS OIORENSIS JUSSIT IOT ESSEM.

34. Au landesmuseum de Darmstadt. (f. Adolf (ioldos(HMid) (op. cit. n. 30). III. p. 29-30. n 87. pl. XXXV.

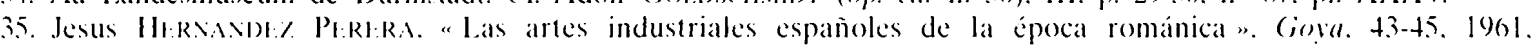
p. 98-112: - Santiago de composede. 1000) ans de pelerinage européen. (jand. 1985. n 24. p. 218-219): PI:TRIS ABBAS MI: JISSI III:RI (dóbut XII" S.).

36. HOC ALTARE SACRI W GEMMIS AI'ROOLE DIECORL $M$

HI:CTI PI:(CATRIX NON RI: SIED VOC BEATRIX

DISPIERIAAT PRORSIS A.NATHIAMAIS IG.NE PIERISITS

OLISOL IS IN ABSTIIIERIT SACRO ('ICTCMOLIE DIC ARIT

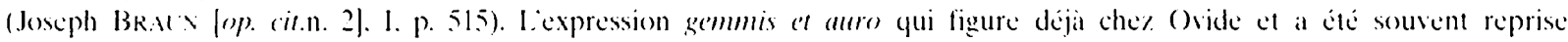
pour des arureses de yualité.

37. MII SI PORIIEI LOCO OI'IS PRORSIS TOLLAT AB IPSO

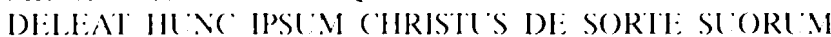

(Joscph BR.UI $\times$ (op. cit. n. 2) 1. p. 465).

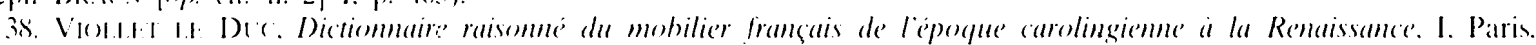

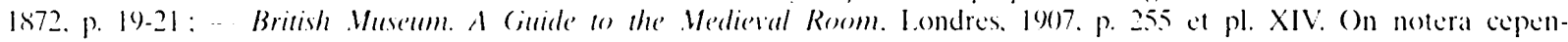
dant que Scheiden a éte d'abord un prieuré de prémontrés. érigé en abbate en 1628 d'apres dom (ontincau. et que Theodoricus I est ke cinquieme "abbe" en 1226 (ciallia christiana. 1. 3. col. 8(12).

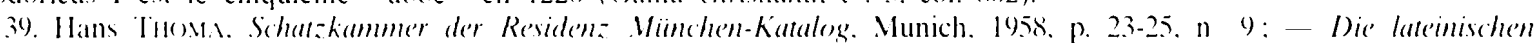

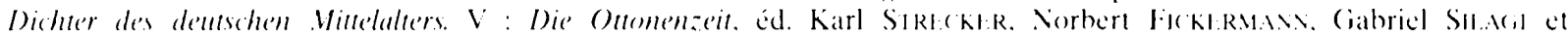

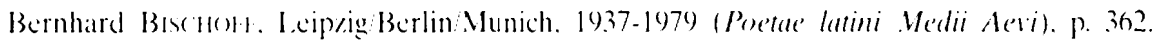


" + Voici César, brillant de sagesse. Henri par son titre

† Christ. il te donnera a honneur. à toi son créateur. ce présent

† dans lequel est enfermée une sainte partie de la croix et la gloire du monde

* Paic-le de retour en lui donnant les joies de la vraie patrie".

Il est vrai qu'il s'agit de l'empereur Henri II le saint (1014-1024). qui a placé son nom en évidence sur toutes les offrandes de grand prix qu'il a faites aux églises de lempire. Au-dessous de la croix gravée sur le cristal était inscrit : CRUX PRECIOSISSIMA. "La croix très précieuse ". et sur la plaque inférieure, autour de l'Agneau de Dieu (AGNUS DEI) on avait donné la liste des reliques de lautel : $\rightarrow$ IN HOC ALTARI SANCTORUM RELIQUIAE CONTINENTUR QUORUM HIC NOMINA SCRIPTA HABENTUR DE LIGNO DOMINI RELIQUIAE SANCTI GEORGII MARTYRIS SANCTI PANCRATII MARTYRIS. SANCTI SEBASTIANI MARTYRIS SANCTI STEPHANI MARTYRIS. SANCTI LAURENTII MARTYRIS DE CRATICULA SANCTI LAURENTII. C"est souvent par le dessous de lautel que l'on introduisait les reliques. comme on le voit dans l'autel de la comtesse Gertrude au muséc de Cleveland. et on trouvera donc à diverses reprises les reliques énumérées à cet endroit. Parmi les saints dont le culte est répandu dans toute la chrétienté on notera saint Pancrace, martyr à Rome en 304. dont les reliques ont été particulièrement vénérées dans l'empire.

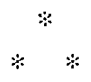

Consécration épiscopale et déposition de reliques étant les éléments canoniques fondamentaux pour un autel portatif, il est normal de trouver quelques inscriptions qui en font état. L'abbaye Saint-Laurent de Liège avait un autel portatif de porphyre. orné d’une bordure de bronze doré sur laquelle on lisait :

ANNO DOMINICAE INCARNATIONIS MIXI INDICTIONE XIV.
IV IDUS AUGUSTI DEDICATUM EST HOC ALTARE IN HONORE
DOMINI NOSTRI JESU CHRISTI ET SANCTAE CRUCIS ET
BEATAE MARIAE SEMPER VIRGINIS ET SANCTI JOHAN.NIS
BAPTISTAE ET OMNIUM APOSTOLORUM ET MARTYRLM
LAURENTII. PANCRATII. GEORGII ET UNDECIM MILLIUM
VIRGINUM ET OMNIUM SANCTORUM COOPERANTE
LAMBERTO II HUJUS LOCI ABBATE MEMORIA DOMINI
HILLINI PRAEPOSITI ET MAJORUM EJUS

Autour de laautel les douze apôtres étaient représentés en ivoire ${ }^{41}$. On a choisi pour la consécration le 10) août. jour de la fête de saint Laurent. patron de l'abbaye. Lambert. abbé de 1061 à 1069 est un lettré qui a notamment écrit une Vie de saint Héribert archevêque de Cologne ${ }^{4 !}$. ce qui est peut-être en relation avec la présence dans l'autel de reliques des onze mille vierges de Cologne. De part et d'autre de la plaque de porphyre rouge d'un des deux autels portatifs du trésor de Conques on a inscrit :

(en haut) ANNO AB INCARNATIONE DOMINI MILLESIMO ( SEXTO KALENDAS JULII DOMNUS PONCIUS BARBASTRENSIS EPISCOPUS ET SANCTE FIDIS VIRGINIS MONACHUS (en bas) HOC ALTARE BEGONIS ABBATIS DEDICAVIT ET DE CRUCE CHRISTI ET SEPUL(CRO) EJUS MULTASQUE ALIAS SANCTAS RELIQUIAS HIC REPOSUIT ${ }^{2}$.

C"est l’époque où le siège de Roda est transporté à Barbastro, avec l'appui de Pedro I ${ }^{\text {er }}$ roi d'Aragon. On savait que Ponce avait été moine de Saint-Pons-de-Thomières, mais cette inscription est le seul document qui le dise moine de Sainte-Foy. L'abbaye de Conques avait plusieurs

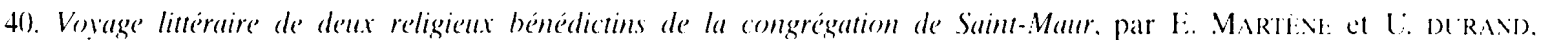
Paris. 1724. p. $189-190$.

41. Histoire littiraire die la France. VIII. Paris, 1868. p. 6-11.

42. C.I.FM. 9. Paris, 1984, p. 41-43, fig. 27-31. Ponce de Barbastro est mort en 1104. ce qui interdit de dater cette consécration du jour des calendes de 1106. 
reliques de la croix. Le cortège des saints qui figure sur les côtés comprend les apôtres et les évangélistes, saint Étienne, sainte Cécile. et trois martyrs agenais. Caprais, Vincent et Foy. Saint Caprais est au centre d’un des longs côtés: le Christ préside à lautre long côté. avec Marie à sa droite. Foy puis Vincent à sa gauche, une hiérarchie qui ne doit rien au hasard. Le trésor de Conques garde d'ailleurs un autre autel portatif. de même date. avec le Christ. entouré de l'alpha et l'oméga. Marie et sainte Foy ti.

Labbaye Saint-Laurent de Liège gardait encore au XVIII' s. un second autel portatif. de pierre verte sertie dans une lame de bronze, qui portait l'inscription de consécration par l'évêque de Liège Raoul le 15 des calendes de mars, en l'an 1137. $5^{\circ}$ indiction. en l'honneur de la Trinité. de la sainte Croix. de la Vierge Marie. de saint Laurent martyr et de tous les saints. Il faut corriger la datation car Raoul de Zähringen a été évêque de Liège de 1167 à 1191. La cinquième indiction se présente pendant son épiscopat en 1172 et 1187: on préférera cette dernière date. parce qu'il suffit dans l'inscription de rajouter un L (MCLXXXVII au licu de MCXXXVII). et que le 15 février 1187 est - si c'est le style de Noël qui a été suivi - un dimanche. jour recommandé pour une consécration. Aux quatre coins de l'autel étaient représentés les quatre évangélistes: sur la plaque inférieure il y avait un crucifix d'ivoire ${ }^{44}$.

Un autel portatif de porphyre vert avec garniture de vermeil est conservé au trésor de la cathédrale de Narbonne. Sur le rebord de la plaque on peut lire linscription suivante :

+ HIC SUNT RELIQUIE SANCTE CRUCIS ET SANCTI ANDREE ET SANCTI BARTHOLOMEI ET SANCTI MATHEI APOSTOLORUM ET SANCTI BLASII ET SANCTORUM JOHANNIS ET PAULI ET SANCTI NICHOLAI ET SANCTARUM VIRGINUM AGNETIS MARGARETE ET BARBARE. HOC ALTARE CONSECRATUM EST ANNO GRATIE MCCLXXIII NONAS FEBRUARII CONSTRUCTUM PER GUIDONEM DE PILEO PRO CUJUS ANIMA CELEBRANTES ORETIS ${ }^{45}$. Le 5 février 1273 est un dimanche: le (jui de Pileo cité en fin de texte doit être celui qui a réalisé l'œuvre (constructum). non l'évêque consécrateur qui. à Narbonne en 1273 est un Pierre de Montbrun: au reste aucun évêque en France ne porte le nom de Gui de Pileo en 1273.

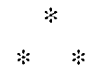

Les inscriptions des autels portatifs de la majeure partie du XI" s. commémorent la croix glorieuse. louent le Dieu très saint. rappellent la vie du Christ.

La croix est au coeur de la vie chrétienne. non la croix de supplice en tant que telle sur laquelle insistera liconographie du Xve s.. mais la croix glorieuse, par laquelle le Christ. en donnant sa vie. rachète l'humanité pécheresse. C"est le sens qüil faut retenir pour l'autel portatif anglo-saxon du deuxième quart du XI" s. conservé au Musée national du Moven Âge à Paris. Autour de la plaque de porphyre ont été gravés en haut le Christ en croix entre les symboles de Lue et de Jean. sur les côtés Marie à la droite du Christ. Jean à sa gauche. et sous chacun d'eux un ange. en bas l'Agneau avec le livre fermé et la croix. entre les symboles de Matthieu et de Marc. Le choix du symbole de Luc. le taureau du sacrifice. figure de la Passion. à la droite du Christ. est évidemment intentionnel : l'autre symbole. l'aigle de Jean. a été placé ici à cause de la proximité de laapôtre figuré sur le long côtéth. Sur les rebords de la plaque des inscriptions commentent liconographie. Au-dessus de la Crucifixion on peut lire:

INGEMIT HIC LU('ASS] PRO RE(iE [JOHA.N.NE]S ET ORA[T]

"Ici l.uc gémit pour le roi. et Jean prie".

4.3. Mhid.. p. 5(1)-51. lig. 26.

4. Vosage lintraire de deux religieux bémédictin... (opp. (it. n. $4(1)$ p. 190 ).

4.5. (I.F.M. 12. Paris. 1988. p. 4?-45. fig. 24-27.

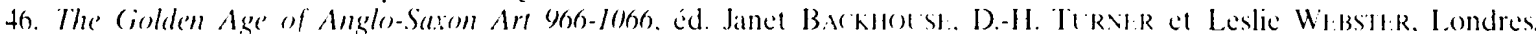

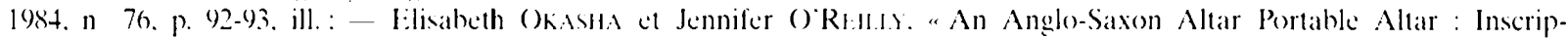
tion and lconography". Joumal of the Harburg and comertauld Institutes, 47. 1984. p. 32-51 (Inscriptions : p. 33-35) 
Le texte peut encore être lu en sa majeure partic. la restitution proposée de Johannes tenant compte de ce qu'il s'agit d’un hexamètre et de la façon dont les deux autres inscriptions conservées sont composées ${ }^{47}$. De la même façon. en effet. les inscriptions des longs côtés commentent les figures de Marie et de l'ange Gabriel :

\section{[GF.]NITRIX MERET GABRIEL CUI SANCTUS ADHERET}

"La mère safflige sur celui auprès duquel saint Gabricl se tient".

DISCIPULUS PLORAT RAPHAEI, QUEM SEMPER ADORAT

"I.e disciple pleure celui que Raphaël toujours adore".

Linscription du rebord inférieur de la plaque a disparu. mais elle devait commenter l'Agneau de l'Apocalypse et l'évangéliste Marc dont le symbole. le lion. est signe de la Résurrection ${ }^{48}$. C"est aussi le sens de l'inscription gravée sur la face supérieure de l'autel portatif de l'abbaye SaintPierre d'Abdinghoff. rapportéc - mais non datéc - par dom Martène et dom Durand ${ }^{40}$ :

IN CRLCE SUSPENSUS AD SE TRAHIT OMNIA CHRISTUS

SURGIT ET INVIC'TUS DE. FUNERE GLORIFICATUS

"Suspendu sur la croix le Christ attire toutes choses à lui.

Il ressuscite de la mort. et invaincu est glorifié».

Le premier vers est la transposition métrique d’une phrase du Christ rapportée par l'Évangile de Jean (XII. 32) : et ego si exaltatus fuero a terra, omnia traham ad meipsum. On retrouve le premier hémistiche dans la littérature du XII s. Le second vers, également léonin, traduit exactement l'affirmation de la croix victorieuse.

L’autel portatif donné par l'empereur Henri II (1014-1024) à la cathédrale de Bamberg. est richement décoré. avec les figures du Christ, de la Vierge. des douze apôtres, et de quatorze séraphins et chérubins ${ }^{5()}$. Linscription est toute tournée vers la louange :

\section{* (HERUBIM QUOQUE ET SERAFIM SANCIUS PROCLAMANT \\ ET OMNIS CELICUS ORDO DICENS : TE DECET I.ACS ET HONOR DOMINE \\ " Chérubins et séraphins proclament qưil est saint \\ et tout l'ordre céleste dit : à Toi. Seigneur. reviennent louange et honneur».}

Les séraphins sont fort peu cités par la Bible, au contraire des chérubins. Le prophète Isaïe. qui leur donne six ailes, les montre glorifiant le Seigneur : et clamabant alter ad alterum et dicebant : Sanctus, Sanctus, Sanctus Dominus, Deus exercituum, plena est omnis terra gloria ejus (Is VI, 3). Il est repris par l'Apocalypse (IV. 8) qui parle des quatre Vivants. portant chacun six ailes et disant : Sanctus, Sanctus, Sunctus, Dominus Deus omnipotens. Linscription renvoie à ces textes bibliques mais plus encore à la liturgie de la messe qui. après la préface et avant le canon, place le triple Sanctus : "Saint. Saint. Saint est le Seigneur. Dieu des armées, les cieux et la terre sont remplis de ta gloire». Le Te decet laus et honor, Domine semble inspiré du Psaume LXV (LXIV). 2 : Te decet hymnus, Deus in Sion "', mais plus encore de l'hymnologie. bien que l'on ne rencontre pas cette expression même, dans les recueils et répertoires d’hymnes du Moyen Âge qui ont des Te decet laus, Te decet hymnus, Te decet omnis gloria, honor, virtus, Te decet laus Domine, Te decet laus, Te decet hymnus, gloriosa Trinitas.

47. Robert Favrat: "Lapport des inscriptions a l'histoire des anges à l'époque romane ". Cahiers de Saint-Michelde-(uxu. 28. 1997. p. 97-98. Cette inscription navait pas été lue de façon complete, mais j’ai pu étudier directement cette lable d'autel grace à la bienveillance de M $^{\mathrm{m}}$ Viviane Huchet. directrice du musée et de Madame Lagabrielle. conservaceur.

48. Robert Fartat. "I.e theme iconographique du lion dans les inscriptions médiévales". Comptes rendus de l'Academie des Inscriptions et Belles-lettres, 1991, p. 613-636.

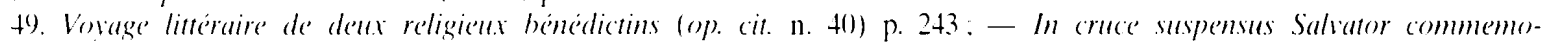
ratur $1 \%$. 2(1) (c)l. 1154).

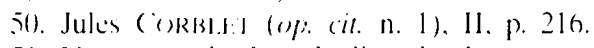

51. Verset repris dans la liturgie du mercredi. Corpus antiphonalium officii. éd. René-Jéan H\&SBERT. Rome, 1968.3. 115315. p. $5(1)$. 
On trouve l'inscription même de l'autel de Bamberg sur un autel portatif d'environ 1180 au Kunstgewerbemuseum de Berlin 52 . et sur un autel portatif de l'église Saint-Michel conservé au musée de Brunswick. on a inscrit un triple Sanctus aussi bien sur la plaque supérieure que sur la plaque inférieure ${ }^{53}$. Sur lautel portatif de Stavelot aux Musées royaux dart et d'histoire de Bruxelles le cristal de roche recouvre un parchemin avec aussi le triple Sanctus du canon de la messe. Comme il s’agit d'exuvres de qualité. destinées à l'usage exclusif de hauts dignitaires ecclésiastiques on peut voir dans ces inscriptions une affirmation trinitaire. comme l'indiquent Grégoire le Grand. Isidore de Séville, Alcuin. Raban Maur. Hincmar. Rupert de Deutz: ou encore Hildebert de Lavardin :

\section{Sanctus, Sanctus, Samctus.}

Ter positum Sanctus, trinum notat esse; sed unnm

Declamat Domimus De'us. Sabatoth...

Quas duo de seraphim nocte dieque canum ${ }^{55}$

Innocent III lui aussi affirmera que "le sacrifice de louange est offert également à l'indivisible Trinité. tant au Père quau Fils et quà l'Esprit de l'un et de l'autre " ${ }^{56}$. Dans cette même tradition patristique se place la plaque inférieure d'un autel portatif du début du x $\mathrm{I}^{\circ}$ s. au Baverische Nationalmuseum de Munich ${ }^{57}$ : au centre. en buste. Dieu bénit el tient un livre. sous la forme du Christ avec nimbe crucifère. tandis qu une inscription dit tout autour :

+ HIC PATER ET LOGOS NECNON PARACLITUS AGiOS

"Ici le Père, et le Verbe et le Saint Paraclet".

l'emploi de mots grees permettant de former, laborieusement un hexamètre léonin.

Cette plaque inférieure de laautel de Munich est cantonnée de quatre bustes de femmes couronnées, dans des médaillons avec leur identification : + JUSTITIA et + TEMPERANTIA en haut, à gauche et à droite. + PRUDENTIA et + FORTITUDO en bas. (es quatre vertus ont été définies par Socrate. Platon. (icéron. Pour le premier. la bonne conduite est fondée sur les vertus de prudence, tempérance, force et justice. pour le deuxième un État correctement fondé est sage, courageux. tempérant et juste, pour le dernier la sagesse nât de la mise en pratique des vertus de prudence. justice. force el tempérance. Elles seront reprises par les auteurs chrétiens. et généralement appelées "vertus cardinales" depuis Ambroise. même si quelques auteurs. Alcuin. Raban Maur. Rupert de Deutz et surtout Thomas d'Aquin. préféreraient l'appellation de "vertus principales" ${ }^{5 \times}$. Katzenellenbogen met en relation les quatre vertus cardinales ainsi placées aux angles de l'autel avec l’autel vétérotestamentaire où quatre cornes étaient disposées aux quatre angles (EX XXXVIII. 2) et cite l'autel de Munich comme la première manifestation de cette iconographie ${ }^{59}$. On peut aussi plus simplement y voir l'attitude vers laquelle il faut tendre quand on s'approche de l'autel. comme l'exprime Sicard de C'rémone à propos précisément de lautel : Altare... est conversatio perfectorum, qui sumt in fide firmi, sapientia fulgidi, quatuor virtutibus eminentes ${ }^{6 t}$. Les quatre vertus cardinales sont placées aux angles de la plaque inférieure d'un autel portatif autour d'un Agneau de Dieu (AGNUS DOMINI) dans un autel conservé au

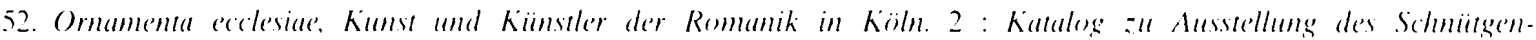

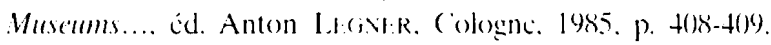

53. Die Inschriften der Stadt Bramschweig bis 1528. n 13. p. 20-21. fig. 12 (1)ie deutschen Inschriften. 35).

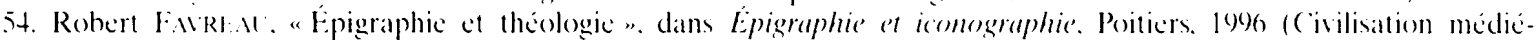
valc. 2), p. 47.

S.) Lersus de msterio missae. Pl. 171. col. 1182

56. De sacro alturis misterio lihri sex. Pl. 217. col. sto

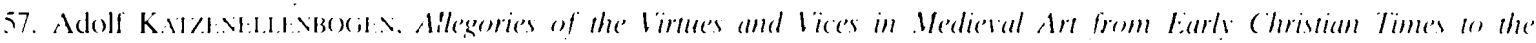

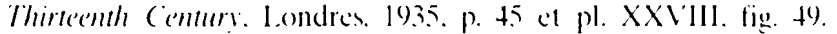

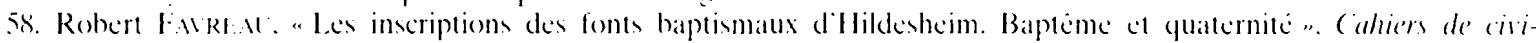
lisation médiévale. XX\III. 1995. p. 119-120.

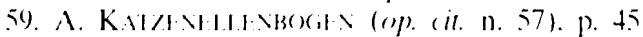

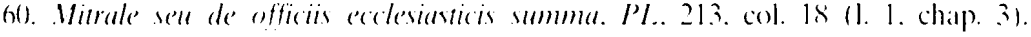


muséc national du Moyen Âge à Paris. denviron $10300^{\text {hl }}$. autour d'une Crucifixion dans un autel du milieu du XII" s. au musée diocésain d'Augsbourg ${ }^{62}$. Elles sont représentées cette fois en pied autour du porphyre vert d'un autel portatif d'environ 1160) au Kunstgewerbemuseum de Berlin ${ }^{6.3}$. Sur la plaque supérieure de l'autel portatif de l'abbatiale St. Peter de Fritzlar. d'environ 1140. les médaillons des symboles des quatre évangélistes, avec les débuts de leurs Évangiles. entourent la pierre d'autel ${ }^{64}$. et sur la face supérieure d'un autel portatif du XII" s. conservé aux Musées royaux d’art et d'histoire de Bruxelles, les angles sont occupés par les médaillons des quatre fleuves du paradis. en haut TIGRIS et GEON. en bas EUPHRATES et PHISON ${ }^{6.5}$. Nous sommes ici renvoyés aux quatre fleuves du paradis que cite la Genèse (II. 10-14). mais on peut rapprocher ces iconographies dautels portatifs, vertus cardinales, évangélistes. fleuves. Saint Jérôme écrit que «les quatre fleuves qui viennent d'une seule source signifient les quatre évangélistes, c’est-à-dire le Christ... Ils irriguent le monde, c'est-à-dire l'Église, et signifient les quatre vertus.... et de même que les quatre fleuves irriguent le paradis. ainsi ces quatre vertus irriguent notre cœur ${ }^{\text {th }}$. ce que reprend exactement une inscription des fonts baptismaux de la cathédrale d'Hildesheim :

$\dagger$ QUATUOR IRRORANT PARADISI FLUMINA MUNDUM

$\dagger$ VIRTUTESQUE RIGANT TOTIDEM COR CRIMINE MUNDUM

" + Les quatre fleuves du paradis irriguent le monde.

$\dagger$ et autant de vertus baignent le cơur pur de péché " ${ }^{67}$.

Le Christ, au centre de l'autel, est la source qui se répand aux quatre points cardinaux du monde par les quatre Évangiles. qui sont comme les quatre fleuves du paradis: les quatre vertus cardinales ont la même source de vie. le Christ. célébré sur l'autel. Il y a là une illustration de la symbolique des nombres familière aux auteurs médiévaux. À propos du nombre quatre. Isidore de Séville rapproche ainsi les quatre évangélistes, les quatre fleuves du paradis, les quatre points cardinaux, les quatre éléments. les quatre vertus cardinales, etc. ${ }^{68}$. Mais l'essentiel est de garder à l'esprit qu'on ne peut commenter l'iconographie d'un autel portatif sans tenir compte de la pierre d’autel sur lequel seront déposés le pain et le vin qui, à la messe. deviendront corps et sang du Christ. Il convient donc que celui qui s’approche de l'autel pour célébrer le sacrifice ait une vie pure. comme le rappelle l'inscription de l'autel portatif d'Orense ${ }^{69}$ et sollicite l'aide du Christ pour communier à ce mystère. comme il est dit dans l'inscription d'un autel portatif de Melk ${ }^{70}$.

Le trésor de la cathédrale de Namur possède un autel portatif du milieu du XI" s. dont l'iconographie est très originale puisqu’il s’agit de dix-huit reliefs sur les côtés illustrant la vie de

61. Jean-Pierre (All11:T, LAntiquité classique, le huut Moyen Âge et Byance an musée de Cluny. Paris. 1985. n 164. p. $239-240$.

62. Anna (. EsmeiJl:R. Divina Quaternitas. A Preliminary Study in the Method and Application of Visual Exegesis. Assen/Amsterdam. 1978, fig. 43: - Jospeh BRAUN (op. cit. n. 2). I. p. 451 et pl. 79.

63. Die Inschriften der Stadt Braunschweig (n. 53) n 12. p. 19-20. fig. 11: - Anton L1:(iNi:R. Romanische Kunst (op). (iit. n. 31). p. 185. ill. 359:- Peter LASkO. Ars sacra (op. cit. n. 29). p. 208: - Patrick M. DI: WINTl:R (op. cit. n. 29). 1985. p. 72:- Heinrich der Löwe und Seine Zeit. p. 234. n D 50.

64. Die Inschriften der Stadt Fritzlar. éd. Théodor Nit:DF:Rot:1.1.. Munich. 1974 (Die deustchen Inschriften. 14), n 3. p. 3-4 : LIBER (iENL(rationis). IN PRINCIPIO VOX (LAMA(ntis). FUIT IN DIEBUSS.

65. Muscés royaux d'ant et dhistoire. Ant chrétien jusquia la fin du mogen âge. catalogue par Ad. Javsi:N. Bruxelles. 1964. n 38. p. 17. pl. XXVI.

66. Pl. 30. col. 533

67. R. Favriat, "Les inscriptions des fonts baptismaux d'Hildesheim" (op. cit. n. 58), p. 116.

68. Liber numeronum qui in sanctis scripturis occurrumt. Pl. 83, col. 183-184. On peut voir aussi létude de Anna ( . F.SWEHII:R (op. cit. n. 62).

69. IESSE DI:(IET ('IARAM VITAM VENIFNTIS AD ARAM...(op. cit. n. 35).

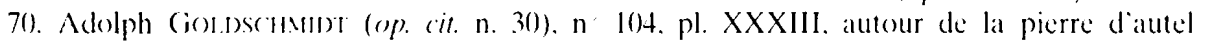

JIIESU (HRISTE: TUI MISTERIA CORPORIS ALMI

DA SUMENDA NOBIS EI CLEMENS SACRA C'RIORIS.

(seconde moitic $\mathrm{x}$ s.). 
Jésus avant sa Passion ${ }^{71}$. La suite débute sur un petit côté et doit se lire dans le sens des aiguilles d'une montre :

ADNUNT[IATIO]. Annonciation. Luc I. 28

MARIA. Visitation, Luc I. 4()

OFFERTORIUM. Présentation au temple. Luc II. 22

DEMONIAC[US]. Guérison d'un possédé. Luc XI, 14

DUO CECI. Guérison daveugles. Matthieu IX. 27

BEATUS VENTER. Glorification de la Mère de Jésus. Luc XI. 27

CLAUDIUS. Guérison d'un paralytique. Luc XXI. 14

CHANANEA. la Cananéenne. Matthicu XV. 22

EPHETA. Gućrison d'un sourd-muet. Marc VII. 34

ASCENDIT. Sermon sur la montagne. Matthicu V. 1

CENTURIO. le Centurion de Capharnaum. Matthicu. VIII. 5

PUELLA SURGE. Résurrection de la fille de Jaïre. Luc VIII. 54

FIMBRIA TA[N] (i[I]T[UR]. Guérison d'une hémorrö̈sse. Luc VIII. 44

SAMARITANA. La Samaritaine. Jean IV. 9

TRANSFIGUR[ATUS]. Transfiguration. Matthicu CVII. 2

DEP[RE]HENSA IN ADULT[ERIO]. la Femme adultère.

QUI SINE [PECCATO]. Jean VIII. 4

CECUS SEDEBAT. l'Aveugle de Jéricho. Marc X. 46

PECCATRIX. le Christ dans la maison de Simon. Luc VII. 37

Les autels portatifs conservés pour le XII" s. sont pour la plupart du domaine de l'empire germanique. Leur iconographie et les inscriptions qui la commentent ont des caractères nouveaux. comme on peut le voir à partir de quelques-uns d'entre eux, particulièrement importants.

L'autel portatif dit d'Eilbert de Cologne ${ }^{72}$, du nom de l'auteur gravé au centre de la plaque inférieure, a fait partic du célèbre trésor des Guclfes: acquis par le musée de Berlin en 1935. il est conservé au Kunstgewerbemuseum. Son iconographie générale est simple : sur les côtés dixhuit prophètes, patriarches et rois. avec sur le rebord inférieur l'inscription suivante :

CELITUS AFFLATI DE (RISTO VATICINATI

HI PREDIXERUNT QUE POST VENTURA FUERUNT

"Inspirés par le ciel ils ont prophétisé sur le Christ.

Ils ont prédit ce qui ensuite allait arriver $"$

Sur la plaque supéricure huit scènes de la vie du Christ et les douze apôtres entourant le Christ en Majesté entre les symboles des évangélistes. avec. sur le rebord. une inscription qui répond à la première :

\section{DOTRINA PLENI FIDEI PATRES DUODENI}

TESTANTUR FICTA NON ESSE PROPHETICA DICTA

"Emplis de la doctrine de la foi les douze pères

témoignent de ce que les paroles des prophètes nétaient pas imaginaires".

On constate au XII' s. un retour à l'Ancien Testament, avec notamment la perspective de démontrer aux juifs qu'il contient les signes annonciateurs du Messic, à une époque où les "disputes" entre juifs et chrétiens ont été particulièrement nombreuses ${ }^{73}$. La seule mise en corrélation entre

71. Ihid. n 61: - Rhin-Meuse. Ant "t cirilisation 80(1)-1200. Cologne/Bruxelles. 1972. p. 223.

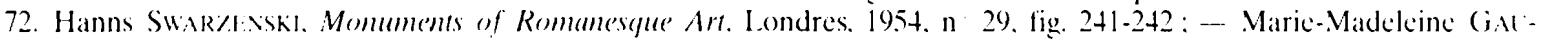

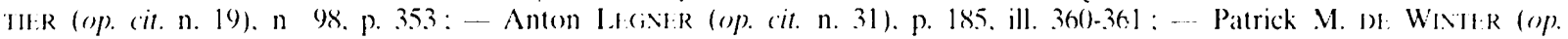
(it. n. 29) p. 65 .

73. Jean-Pierre (All.til. "I a réappropriation du prophétisme par les imagiers chrétiens du XIle siecte". Le monde de la Bible. n 131. 200\%. p. 47-53: - Robert FArRI:Al. "Controverses judéo-chrétiennes et iconographie. Lapport des inscriptions". Comples rendus de lacudémie des inscriptions et belles-lettres. 2001. p. 1267-13013. 
prophètes et apôtres était déjà significative. Dans le cas de lautel d'Eilbert de Cologne chaque personnage vétérotestamentaire et chaque apôtre tiennent un phylactère avec un texte. Que l'on ait ainsi ajouté vingt-neuf textes à une iconographie déjà abondante, sur un autel portatif de dimensions modestes par nature $-13.3 \times 35.7 \times 20.9 \mathrm{~cm}-$ implique que l'on prenne en compte l'apport épigraphique pour le commentaire de cet autel. Un apport savant parce que destiné à un prélat, donc quelqu'un de grande culture. Un apport de grande cohérence pour les apôtres, mais complexe pour les prophètes.

Les deux textes des bords inféricur et supérieur commencent au début d’un même long côté. Le premier prophète qui corresponde à cette situation est Daniel qui tient un phylactère sur lequel on lit : (UM VENERIT SANCTUS SANCTORUM (ESSABIT UN[C]TIO). "Lorsque viendra le saint des saints l'onction cessera". Le texte est inspiré de Daniel IX. 24 (et ungatur sanctus sanctorum) qui introduit la célèbre prophétie des soixante-dix semaines ${ }^{74}$. interprétéc au Moyen $\hat{A}$ ge comme lannonce de la date de la venue du Messic. de sa mort et de la destruction du temple en 7(). et constamment opposée par les auteurs chrétiens aux juifs lors des "disputes" comme une preuve incontestable de la venue du Sauveur. La citation même de Daniel est tirée du "Sermon contre les païens. les juifs et les ariens " de Quodvultdeus, évêque de Carthage au début du $v i$ s. un texte constamment repris au XII s. dans les controverses judéo-chrétiennes et dans les drames liturgiques. avec de nombreuses représentations iconographiques ${ }^{75}$. À partir de la naissance du Christ. il n'y a plus de roi oint en Israël. Les citations d'Isaie : ECCE VIRGO CONCIPIET [ET] PARIET FILIUM (VII, 14) : "Voici que la Vierge concevra et elle enfantera un fils " et de Jérémie. VISUS EST IN TERRIS ET CUM HOMINIBUS CONVERSATUS EST (Baruch III. 38). "Il est apparu sur la terre et a conversé avec les hommes". sont aussi celles que l'on trouve dans le sermon où Quodvultdeus fait défiler devant les juifs des personnages de l'Ancien Testament qui ont annoncé la venue du Christ ${ }^{76}$. Comme dans les discussions entre chrétiens et juifs, ces derniers objectaient que la phrase était de Baruch - quils ne reconnaissaient pas - et non de Jérémie. Pierre de Blois leur rappellera que Baruch avait reçu ces mots de la bouche de Jérémie car il était son notaire ${ }^{77}$.

Éréchiel est le seul des quatre grands prophètes qui ne soit pas cité par Quodvuldeus. Venant après Danicl il dit : SANCTIFI(ABO NOMEN MEUM QUOD POLLUTUM EST INTER (jENTES (XXXVI. 23). "Je sanctifierai mon nom qui a été profané parmi les nations", et le verset se termine par : "et les nations sauront que je suis le Seigneur quand je ferai éclater ma sainteté sous leurs yeux". ('e verset élait bien adapté à la messe. célébration du Christ mort et ressuscité. Après lui David présente le verset 12 du Psaume XCIV (XCIII) : BEATUS EST QUEM TU ERUDIERIS DOMINE. "Heureux celui que tu instruis. Seigneur ». Ce Psaume était lu aux matines du vendredi dans la liturgie des heures (bréviaire) ${ }^{78}$. Melchisédech vient ensuite tenant le pain et le vin. "Melchisédech. roi de Shalem. apporta du pain et du vin. il était prêtre du Dieu Très haut" (Gn XIV, 18), et l'épître aux Hébreux appliquera au Christ la parole du Psaume (CX, 4) : "Tu es prêtre pour l'éternité selon l'ordre de Melchisédech ". Le célébrant devait avoir sous les yeux la figure de Melchisédech - rappelée au canon de la messe - au centre du long côté de l'autel portatif. Le prophète suivant. Osée. annonce que "le nombre des fils d'Israël sera comme le sable de la mer». ERIT NUMERUS FILIORUM IS[R]AHEL QUASI ARENA MARIS (I. 10). On ne voit pas d'évidence pourquoi cette citation sur un autel. Dans son commentaire sur le livre d'Osée. saint Jérôme dit que le nombre des fils d’Israël sera. comme le sable de la mer. dispersé dans tout l'univers: alors Israël. aujourd hui captif. se recon-

74. E. PIII.IPp1., "Daniel (le livre de)". dans Dictionnaire de la Bible. II. dir. F. Vicioltrolx. Paris. 1910, col. 12771282 (Révélation des soixante-dix semaines).

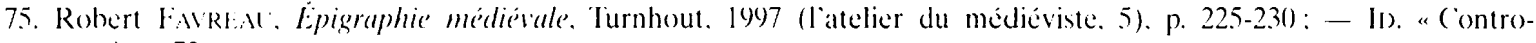
verses..." (ci. n. 7.3).

76. La seule différence par rapport au texte latin de la Bible est linversion Visas est in terris au lieu de In terris visus est. Le choix de Baruch pour Jérémic lail pencher pour une rélérence au Scrmon de Quodrultedeus.

77. Pl. 207. col. 849. Il est effectivement dit dans Jérémie: Scripsit Baruch ex ore Jeremiae (XXXVI. 4).

75. (ompus antiphomaliem officii. IV. ¿de. Renci-Jean H1:SB1:RT. Rome. 1970. n 6212. p. 55. 
naîtra un chef. et ce sera le grand jour du Christ ${ }^{79}$. La prophétie de Malachie peut se suffire à elle-même : VOBIS TIMENTIBUS DEUM ORIETUR SOL JUSTITIAE (IV. 2). "Pour vous qui craigne\%. Dieu se lèvera le soleil de justice». Elle est retenue par labbesse Herrade dans son Hortus deliciarum.

Au milieu de l'autre long côté ont été placés Isaïe el Jérémie. avec leurs textes. cités notamment par Quodvultdeus. qui annoncent la venue du Messie. À gauche sont deux petits prophètes. Jonas et Nahum. La prophétic du premier dit : TOLLE ANIMAM MEAM QUONIAM MELIOR EST MI[HI] MORS QUAM VITA (IV. 3). "Prends ma vie car mieux vaut pour moi mourir que vivre». Jérôme lapplique au Christ qui en sa vie n’a pu sauver une seule nation. Israël. et par sa mort a sauvé le monde ${ }^{80}$. Peut-être aussi l'auteur du programme a-t-il eu en pensée la phrase de Paul dans l’épître aux Philippiens (1. 21) : mihi enim vivere Christus est. "t mori lucrum. "pour moi certes vivre cest le Christ et mourir mest un gain". Sur le phylactere de Nahum on lit : REDDIDIT DEUS SUPERBIAM JACOB SICUT SUPERBIAM ISAREL (II. 2). "Dicu a rendu la fierté à Jacob comme la fierté à Israël». Étant donné la corrélation faite entre prophètes et apôtres. on peut ici penser au commentaire de Rupert de Deutz. un presque contemporain et proche de Cologne : "sous les noms de Jacob et d'Israël sont compris tous les élus. tant anciens que nouveaux. tant ceux qui ont précédé que ceux qui suivent la venue du (hrist. Car nous recevons Jacob comme le pere selon la chair de douze fils et dautant de tribus disraël. et le Christ comme père. selon l'esprit, des douze apôtres et de tous ceux qui par eux ont cru et croient en lui " ${ }^{\$ 1}$. Sur la partie droite de ce long côté le texte de Salomon ne pose pas de difficulté : PER SAPIENCIAM SANATI SUNT QUI PLACUERINT DOMINO A PRINCIPIO (Sg IX, 19), "par la Sagesse ont été guéris ceux qui ont plu au Seigneur dès l'origine». En revanche le texte de Joël est pour le moins obscur : COMPUTRUERUNT JUMENTA IN STERCORE SUO (1. 17), "Les bêtes de somme ont pourri sur leur fumier " ${ }^{2}$. Le texte a été peu commenté par les exégètes. du fait sans doute de sa difficulté. Le contexte de la citation est un contexte de désolation : bêtes mortes, granges dévastées. greniers en ruines car le blé fait défaut. Saint Jérôme écrit que pourrit sur son fumier celui dont le dieu est son ventre: pour lui sont démolis les granges de la félicité future. les greniers de l'abondance éternelle. et les pressoirs sont renversés. car si froment et vin manquent. c'est en vain que granges et pressoirs sont préparés ${ }^{83}$. On rejoint ici la signification de l'autel, où sont les nécessaires pain et vin de vie. Pour Rupert de Deutz. les bêtes qui pourrissent sur leurs fumiers sont ceux qui finissent leur vie dans la luxure: le sens du texte est : à toi. Scigneur, je confesse mes péchés, à cause de nos péchés nous sommes livrés en la main d’ennemis qui ont dévasté et brûlé notre terre ${ }^{\$ 4}$.

Les textes des petits côtés ne sont guère plus explicites dans leur relation avec lautel. (On peut comprendre la parole de Jacob : VIDI DOMINUM FACIE AD FACIEM (Cin XXXII. 30), "Jai vu le Seigneur face à face". qui se poursuit dans le verset : "et jai eu la vie sauve": cest un texte quemploie la liturgie du deuxième dimanche de Carême ${ }^{85}$.

La citation d’Abdias est. quant à elle. particulièrement obscure : TRANSMIGRATIO JERUSALEM QUE EST IN BOSPHORI POSSIDEBIT CIVITATES AUSTRI (verset 20). "Les exilés de Jérusalem qui sont dans le Bosphore posséderont les cités du midi ». Pour saint Jérôme. l'exil a lieu à Sepharad. quil traduit par Bosphore. ou encore dans toutes les régions. les cités du Midi sont dans la tribu de Juda: dans un sens spirituel l'exil des fils d'Isrä̈l cest l'abandon par eux

79. Pl. 25. col. 829 .

8). Ibid.. col. $11+6$.

81. Commenturii in duodecim prophetas minoris. Pl. 168. col. 549.

82. I a Bible de Jérusalem qui traduit : "L.es grains se sont racornis sous leurs motles" (?). dit : "sems incertain" . "t la T.(O.B. : "Ie's grains sont desséchés sous la glibe" dit de méme : "vers diflicile, sur quatre mots trois ne se trouvent qu ici".

8.3. (commenturius in Joelem prephetem. Pl, 25. col. 960).

8t. In Jö̈l, Pl. 168. col. 213.214.

85. Corpus amliphomalium officii (n. 78). n 7874. p. 4h0). On notera que lat reference de la citation we (in XXXII. 31 dans la Bible de Jérusalem. 
de la lettre qui tue pour l'esprit qui vivifie. de la superbe des juifs pour l'humilité chrétienne. les églises de la vraie el parfaite lumière ${ }^{\text {st. }}$. Pour Rupert de Deutz. le Bosphore est une région de la mer septentrionale que ne mentionne pas l'Écriture: les nations païennes qui étaient sans connaissance de Dieu, dans l'obscurité et le froid, posséderont les cités du Midi, c'est-à-dire la plénitude de la vraie lumière et de la chalcur. grâce à Dieu qui. par sa seule grâce, a visité les nations ${ }^{\$ 7}$. Le Seigneur. célébré sur l'autel. est donc celui qui apporte aux nations la vraie lumière. Le texte du dernier prophète de ce petit côté. Zacharie. QUI TETIGERIT VOS TANGET/PUPILLAM OCULI MEI (II. 8), "Qui vous aura atteint m'atteindra à la pupille de l'œil ". $s$ 'entend de la protection du Seigneur pour ses fidèles contre toutes les parties adverses 88 .

Sur le dernier petit côté. un des prophètes a disparu. et il ne reste que Sophonie et Balaam. MANE. MANE JUDICIUM SUUM DABIT DOMINUS IN LUCEM ET NON ABSCONDETUR dit le premier (III. 5). "Matin après matin le Seigneur promulguera son droit. et il ne fait pas défaut ". Le Seigneur est le juste juge. il s'efforce d’appeler la cité corrompue à se convertir. mais l'inique Israël n a pas reconnu son erreur ${ }^{89}$. L'oracle de Balaam. EX JACOB STELLA PRODIET ET DE ISRAHEL HOMO SURGET (Nb XXIV, 17), "Un astre issu de Jacob surgira et un homme se lèvera d'Israël». est évidemment une annonce de la venue du Messie. ce qu a retenu la liturgie qui. comme ici a remplacé le consurget virga par exsurget homo, et cite ce verset à deux reprises pendant l'Avent et à l'Annonciation ${ }^{\%}$.

Il est fortement question dans ces textes de la supériorité de la foi chrétienne sur la loi d'Israël. mais le choix des prophéties est souvent très original, voire obscur sans l'éclairage de commentaires peu nombreux sur les petits prophètes. On peut se demander sil n'y a pas dans le milieu de Cologne une source qui donnerait une clé de lecture générale de ces dix-sept textes. car une partie de ceux-ci se retrouvent dans des ouvres du même milieu et de même époque. C"est le cas en particulier du reliquaire coupole de Hochelten au Victoria and Albert Museum de Londres, une cuvre de Cologne, vers 118(), où sont mis en correspondance prophètes et apôtres ${ }^{91}$. Sur les dix textes des phylactères des prophètes. six sont les mêmes que ceux de l'autel portatif d'Eilbert de Cologne. ceux d'Isaïe (VII. 14) et de Jérémie (Ba III. 38). mais aussi ceux de Joël. Abdias. Sophonie. Jonas qu'on ne retrouve pas. à ma connaissance. dans d'autres xuvres. Dans le même musée est conservé un prophète Zacharie qui a la même citation que sur laautel d'Eilbert: il provient d'une tour reliquaire des martyrs de la légion thébaine et des onze mille vierges martyres conservée au Hessisches Landesmuseum de Darmstadt. couvre du maître de lautel de saint Grégoire de Siegbourg pour une église de Cologne. vers $1165 / 75^{12}$. On peut encore joindre au dossier une enluminure du lectionnaire de l'archevêque Frédéric de Cologne. vers 1130 , où l'on trouve pour Ézéchiel et Malachie les mêmes citations que sur l'autel portatif d'Eilbert ${ }^{\prime 3}$. et la châsse de saint Héribert au trésor de l'abbaye de Deutz pour la citation de Malachie ${ }^{94}$.

Au centre de la plaque supérieure le Christ en Majesté. trône en bénissant. entouré d'une mandorle et des symboles des évangélistes. Tout autour de lui les douze apôtres tiennent chacun. sur un phylactère. une phrase du "symbole des apôtres". À gauche quatre petits tableaux représentent l'Annonciation. la Visitation. la Nativité. la Présentation au temple, à droite quatre autres

86. Commentarius in Abdiam prophetam, Pl. 25, col. 1115-116.

87. In Abdias, Pl. 168. col. 394.

88. Commentarius in Zachariam prophetam de saint Jéróme. PL. 25. col. 1433.

89. Commentarius in Sophoniam prophetam de saint Jérôme, PL. 25. col. 1373-1374: In Sophoniam prophetam de Rupert de Deut\%. PL, 168. col. 674.

9). (orpus antiphonalium officii (op. cit. n. 78), n 73338 . p. 3.34.

91. Annales archéologiques, 19. 1859, p. $13 ; 22.1862$. p. $5: 24.1864$, p. $11: 25.1865$, p. $5.69:-$ Hermann S(HNT\%IIR. Rheinische Schatzammer. Die Romamik. II. Düsseldorf. 1959. n 17. p. 25. pl. 6.3-67.

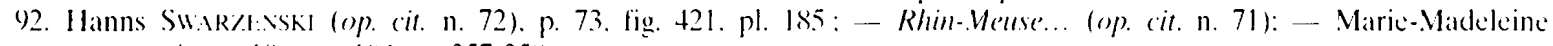
(i.M IHIt:R (op. (it. n. 19). n 104. p. $3.57-3.58$

93. Rhin-Meuse (op. cit. n. 71). p. 30)8. pl. 541 (ms. 59 de la bibliotheque de la cathédrale de (ologne).

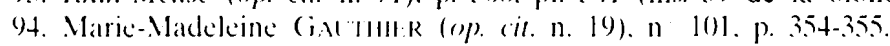


tableaux illustrant la Crucifixion. les Saintes femmes au sépulcre. la Descente du Christ aux enfers. l'Ascension. La lecture des inscriptions se fait dans le sens des aiguilles d'une montre :

\author{
PETRUS DIXIT : CREDO IN DEUM PATREM OM(nipotentem) CRE(atorem) C(acli E(t) T(errac) \\ ANDREAS DIXIT : ET IN JHESUM CHRISTUM FILIUM EJUS U(nicum) D(ominum) N(ostrum) \\ JACOBUS DIXIT : QUI CONCEPTUS EST DE S(piritu) S(ancto) N(atus) EX M(aria) V(irginc) \\ JOHANNES DIXIT : PASSUS SUB PONCIO PILATO ((rucifixum) M(ortuus) ET S(epultus) \\ TOMAS DIXIT : DESCENDIT AD INFERNA TERCIA D(ic) R(esurrexit) A [mortuis] \\ JACOBUS DIXIT : ASCENDIT AD CELOS SEDET A(d) D(exteram) P(atris) O(mnipotentis) \\ PHILIPPUS DIXIT : UNDE VENTURUS EST JUDICARE V(ivos) E(t) M(ortuos) \\ BARTHOLOMEUS DIXIT : CREDO IN SPIRITUM SANCTUM S(anctam) E(cclesiam) C(atholicam) \\ MATHEUS DIXIT : SANCTORUM COMMUNIONEM \\ SIMON DIXIT : REMISSIONEM PECCATORLM \\ TATHEUS DIXIT : CARNIS RESURRECTIONEM \\ MATHIAS DIXIT : ET VITAM ETRENAM AMEN.
}

Le symbole est attribué aux apôtres dès le II $s$. (saint Irénée). Rufin, vers 400). est le premier témoin de l'attribution à chacun des apôtres d'un article du symbole : "Nos anciens rapportent quaprès l'Ascension du Seigneur. lorsque le Saint Esprit se fut reposé sur chacun des apôtres sous forme de langue de feu afin qu'ils pussent se faire entendre en toutes les langues, ils reçurent du Seigneur l'ordre de se séparer et d'aller dans toutes les nations pour prêcher la parole de Dieu. Avant de se séparer ils établirent en commun une règle de la prédication qu'ils devaient faire. afin que, une fois séparés. ils ne fussent pas exposés à enseigner une doctrine différente à ceux quiils attiraient à la foi du Christ. Étant donc tous réunis et remplis de l'Esprit Saint ils composèrent un bref résumé de leur future prédication. mettant en commun ce que chacun pensait, et décidant que telle devra être la règle à donner aux croyants. Pour de multiples et très justes raisons ils voulurent que cette règle s'appelât symbole " ${ }^{95}$. Cette croyance fut admise au $v^{\prime}$ s. par Maxime de Turin. Cassien. Fauste de Riez, au vIr s. par Isidore de Séville. Ildefonse de Tolède. Leétat ancien du symbole comprenait douze articles. ce qui a dû conduire à attribuer un article à chaque apôtre au $V^{\circ} \mathrm{s}$. Le texte reçu qui figure dans la liturgie du bréviaire, du rituel, etc. comporte des premiers articles plus développés et deux articles supplémentaires, d'où des variantes dans les différentes auvres qui présentent chaque apôtre avec un article du symbole, selon les regroupements de texte choisis. Neil Stratford a mis en parallèle huit ocuvres des XII" et XIII" s. qui présentent les apôtres avec l'article du symbole qu'ils ont choisi ${ }^{96}$. Pierre. André. Jacques et Jean sont toujours en tête. Simon Jude et Mathias, pas toujours en cet ordre, sont les derniers cités. L'ordre suivi dans l'autel d'Eilbert de Cologne est celui du canon de la messe. à la réserve quil ny a pas Paul, cité au canon avec Pierre. et que Mathias ne figure pas au canon. Le choix du symbole des apôtres autour de la partie essentielle de l'autel quest la pierre sur laquelle sont placés patène et calice marque bien la supériorité de la Nouvelle Alliance sur l'Ancienne représentée par les prophètes. À la même époque l'abbé de Saint-Denis Suger l'exprime parfaitement en une courte formule dans une des verrières de son abbatiale :

\title{
QUOD MOYSES VELAT CHRISTI DOCTRINA REVELAT
}

"Ce que Moïse voile, la doctrine du Christ le dévoile " ")

ce qu'Augustin disait déjà : In veteri Téstamento est occultatio Novi et in Novo Testamento est manifestatio Veteris ${ }^{\text {g). }}$.

95. Commentarins in symbolum apostolorim. PL. 21. col. 337: - A. VAcAst. "Apotres (le symbole des"). dans Dictionnaire de theologie catholique. II Paris. 1909. col. 1660)-1680).

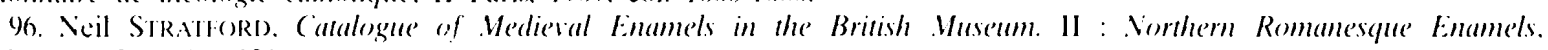
Londres. 1993. p. 119-121.

97. Sicier. Eunres. 1. éd. Françoise (insparro. Paris. 1996 (l.es (lassiques de lihistoire de france au Moven Áge. 37). p. 148. De meme à la façade occidentale de Saint-Trophime d'Arles : LEX MOISI (LLAT QLOD PALLI SIERMO) RIEVIIAT. C.I.F.K. 1. 14. 1989. p. 42-43.

9s. Pl. 40. col. 315. 
Un des autels portatifs du trésor de l'église St. Servatius de Sicgburg dit de Saint-Maurice ${ }^{99}$. de la fin du XII" s.. montre la même disposition des dix-huit grands et petits prophètes sur les côtés et des douze apôtres autour de la pierre dautel en porphyre. les premiers avec leur nom sur un phylactère. les seconds avec des livres fermés - sauf Pierre qui porte les clés - sans inscription. Ine impressionnante liste de reliques (HIC CONTINFNTIJR REIIQUJAF) est énuméréc sur les bords de la plaque inférieure ${ }^{100)}$. Mais, plus que la mise en concordance de l'Ancien et du Nouveau Testament. c’est l'iconographie de la plaque supérieure qui retient l'attention. À gauche sont représentées la Trinité et la Passion (PASSIO CHRISTI), à droite l'Apparition du Christ ressuscité à Maric Madeleine. les Saintes femmes au tombeau vide (SEPULCRUM DOMINI). et l'Ascension. La référence est on ne peut plus claire au canon de la messe où le prêtre, aussitôt après la consécration, fait mémoire de la Passion, de la Résurrection et de l'Ascension. les mots mêmes inscrits sur l'autel portatif : Unde et memores, Domine, nos servi tui, sed et plebs tua sancta, ejusdem Christi Filii tui, Domini nostri, tam beate passionis, necnon et ab inferis resurrectionis, sed et in caelos gloriosae ascensionis, offerimus... panem sanctam vitae aeternae et calicem salutis perpetuae. On se souviendra ici particulièrement que l'iconographic d'un autel portatif doit se comprendre en fonction des Saintes espèces placées sur la pierre dautel. Les apôtres sont ici l'Église appelée à poursuivre l'œuvre du Christ après son Ascension. Deux idées supplémentaires sont soulignées par l'iconographie et l'épigraphic. À la partie gauche de la plaque le sang coule des pieds du Crucifié jusque sur le corps d'Adam qui sort du tombeau. Dès le $H^{\circ}$ s. Origène place la sépulture d’Adam au Calvaire, et après lui saint Ephrem. saint Jean Chrysostome, saint Athanase. En Occident la même croyance a cours à partir de la fin du IVe s. : Carmen adversus Marcionem ${ }^{101}$, traité de saint Ambroise sur l'Évangile de Luc ${ }^{102}$. sermon de saint Césaire un peu plus tard ${ }^{103}$. Déjà saint Paul écrivait aux Corinthiens : "de même que tous meurent en Adam, ainsi tous revivront dans le Christ" (1 Cor XV. 22). Tertullien appellera le Christ Adam novissimus, Maxime de Turin Adam novus. Jérôme ou Paulin de Nole. Adam secundus ${ }^{104}$. Ln crucifix de l'église Saint-Michel de Lunebourg a la même iconographie que l'autel de Saint-Maurice. et sous Adam qui sort du tombeau une inscription dit ${ }^{105}$ :

\section{ADAE MORTE NOVI REDIT ADAE VITA PRIORI}

"Par la mort du nouvel Adam la vie revient au premier Adam".

Un tableau réemployé pour servir de reliure. œuvre d’un maître colonais vers 1180 ), dit de même ${ }^{106}$ :

\section{$\doteqdot$ QUOD VETUS EXEMIT NOVUS ADAM A MORTE REDEMIT \\ SUSCITAT INDE DEUS CORRUIT UNDE REUS \\ VITA REDIT MORS VICTA PERIT HOMO SURGERE CREDIT \\ SUMMAQUE CUM DOMINO SCANDERE REGNA SUO}

« $\dagger$ Ce que le vieil Adam a perdu, le nouvel Adam le racheta par sa mort.

Dieu suscite le salut d'où le coupable avait tiré la chute.

La vie revient. la mort vaincue périt. l'homme croit qu'il ressuscitera

Et montera avec son seigneur aux royaumes suprêmes".

La présence de la Synagogue et de l'Église rappelle le contexte des controverses judéo-chrétiennes du XII s. Ainsi, c’est toute l'humanité qui est sauvée et comme recréée par le sacrifice du Christ. nouvel Adam, rappelé sur l'autel. En second lieu. la représentation de la Trinité. Père

99. Sur la plaque inférieure une inscription (postérieure) disait : Hoc aitare vere sancti Mauritii martiris est et ducis gloriosi.

J(O). Clemens . M. M. BAYIR. "Der Mauritius-Tragaltar in Sieghurg : Bemerkungen zu Datierung. Ikonographie und Ikonologie unter besonderer Berücksichtigung der Inschriften". Heimathläter des Rhein-Siegkreises. 60/1. 1992/93. p. 7-46.

101. PL. 2. col. 1067-1068) (attribution erronéc à Tertullien).

102. Traité sur l'Évangile de Luc. $2^{c}$ éd.. dom (Gabriel TISSO)T. Paris. 1958 (SC. 52). p. 194.

103. Pl. 39. col. 1751 (attribution erronéc à Augustin).

104. Pl. 2. col. $869: 57$. col. 239. 249. 570:23. col. 328 .

105. DiDRon. "Bronzes et orfêvrerie du Moven Âge". Annales archéologiques. XIX. 1859. p. 88-89.

106. Marie-Madeleine (in tumle (op. cit. n. 19). n 103. p. 356 (tableatu avec 16 figures de la Passion). 
- sous les mêmes traits que le Fils et avec un nimbe crucifère -. Saint-Esprit sous la forme d'une colombe. et Fils sur la croix. avec le mot : TRINITAS. affirme avec force que le sacrifice rédempteur est sacrifice de l'indivisible Trinité. même si le Fils seul est représenté sur la croix ${ }^{107}$. Lautel portatif de Stavelot ${ }^{101}$. vers 1150/60), conservé au Musées royaux d'art et d'histoire de Bruxelles. est une ceuvre illustrant la Mort et la Résurrection du Christ. annoncées par les figures de l'Ancien Testament. célébrées dans l'eucharistie. et aussi une ouvre se situant dans la polémique anti-juive. avec la Synagogue, à la fois "aveugle " à l'enseignement des prophètes. et représentant les juifs coupables de la mort du Christ. En haut et en bas de la plaque supérieure six tableaux sont consacrés à la Mort et à la Résurrection du Christ. en bas la Cène (CENA). la Comparution devant Pilate (PILATUS. JUDEI). la Flagellation (FLAGELLATIO) DOMINI). en haut le Christ portant la croix (BAJULANS CRUCEM CHRISTUS). la Crucifixion (S. MARIA. S. JOHANNES). le Tombeau vide(ANGELUS. MULIERES - RESURRECTIO DOMINI). Le terme de bajulans est ici un rappel direct de Luc (XIV. 27) et de Jean (XIX. 17). Laannonce de la Passion est faite par quatre scènes de l'Ancien Testament. aux quatre écoinçons déterminés par le quadrilobe qui entoure la pierre d'autel en cristal de roche. La première est le Sacrifice d'Isaac par Abraham (IMMOLATIO ISAAC). avec Isaac qui porte le bois. comme. dans la tableau le plus proche. le Christ porte sa croix (Gn XXII). Dès les premiers auteurs chrétiens. Isaac a été vu dans cette scène comme une figure du Christ. Tertullien écrit dans son Adversus Marcionem : Isaac, cum a patre hostiam deditus, lignum sibi ipse portaret, Christi exitum jam denotabat, in victimam concessi a Patre et lignum passionis bajulantis. ${ }^{109}$. Après lui Zénon. Augustin. Prosper d'Aquitaine. Léon le Grand. Maxime de Turin. Paulin de Nole. Eugyppius, Raban Maur, Lanfranc. Bruno. Rupert de Deutz. Hildebert de Lavardin. Adam Scot reprendront la même comparaison. La deuxième scène est celle du serpent d'airain dressé dans le désert par Moïse (Nb XXI. 9) : "Moïse fit un serpent d'airain et le pendit à un poteau. et si les serpents mordaient quelquiun et que celui-ci regardait le serpent daairain. il demeurait en vie ». Le Christ lui-même a donné ce signe comme annonce de sa mort sur la croix : "De même que Moïse a élevé le serpent dans le désert, ainsi faut-il que le fils de l'homme soit élevé" (Jn III. 21). Saint Ambroise ${ }^{110}$ et d’autres après lui rappelleront cette figure vétéro-testamentaire de la croix. Moïse est identifié par les tables de la Loi (LEX DOMINI). La troisième scène est celle de l'Offrande de l'Agneau par Abel (HOSTIA ABEL), la quatrième l'Offrande du pain et du vin par Melchisédech, cette dernière placée immédiatement au-dessus de la dernière Cène. Bien des auteurs ont vu dans Abel une figure du Christ, victime innocente. et dans Melchisédech une figure du Christ, grand prêtre par excellence. intermédiaire entre Dieu et les hommes. Mais ici. plus qu'un rapport patristique. il est fait référence au canon de la messe où le prêtre demande au Seigneur d’accepter l'offrande de l'autel "comme il a accepté les présents d'Abel le juste. le sacrifice du patriarche Abraham. le sacrifice du grand prêtre Melchisédech».

L'annonce de la Résurrection est faite par les deux scènes latérales incluses dans la quadrilobe. à gauche Samson enlevant sur son épaule les portes de Gaza, à droite Jonas sortant de la bouche du poisson. l'un et l'autre accompagnés de leur nom. La figure de Samson se libérant de sa prison et emmenant avec lui les portes de Gaza n'est pas souvent représentée dans l'art. mais elle donnée par les commentateurs du chapitre 16 du livre des Juges comme une figure du Christ ressuscité. ainsi de Raban Maur ${ }^{111}$. que recopie à trois ou quatre mots près Rupert de Deutz ${ }^{112}$. Gaza signifie l'enfer. les Philistins les juifs, dont la "perfidie " 113 est démontrée. eux qui ont placé des gardes au tombeau du Christ. Samson qui. au milieu de la nuit. sort de Gaza

107. (f. ce qui a éle dit ci-dessus à propos du triple Sanctus sur plusieurs autels portatifs.

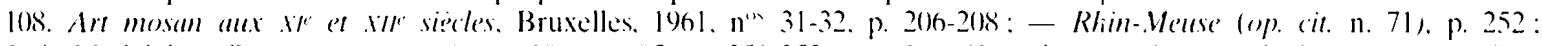

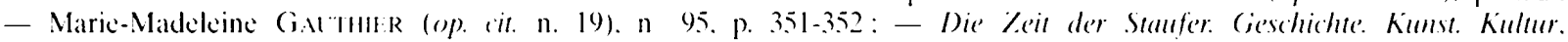

éd. Reiner Halsshtrr. I. Stutgart. 1977. p. 409-411: Peter I.ASKO (op. (it. n. 29). p. 197-198. fig. 269.

109. Pl. 2. col. 3+6.

110. Ihid.. 17. col. 695.

111. Ihid.. 108. col. 1194 (Commentaria in librum Judicum).

112. Ihid.. 167. col. 1049-1050) (In librum Judicum commentarium liber anus).

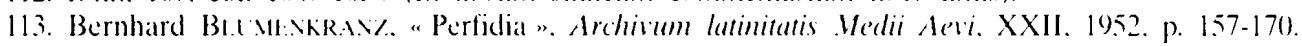


et en emmène la porte jusqu'au sommet de la montagne, c'est le rédempteur, qui ressuscite avant l'aube. sort libre de l'enfer. en brise les portes et monte aux cieux. Quant à Jonas, il est envoyé par Yahvé à Vinive: au cours de sa traversée une tempête se lève, les matelots y voient la faute de Jonas et le jettent à la mer, un grand poisson l'engloutit, "et Jonas resta trois jours et trois nuits dans les entrailles du poisson», puis fut rejeté sur la terre ferme ${ }^{114}$. Le Christ luimême a donné "le signe de Jonas" comme l'annonce de sa mort et de sa résurrection : " comme Jonas resta dans le ventre du poisson trois jours et trois nuits, ainsi le Fils de l'homme restera dans le sein de la terre trois jours et trois nuits ${ }^{115}$. Bien des exégètes naturellement commentent par la suite le "signe de Jonas »: Jonas envoyé prêcher à Ninive, c'est le Christ envoyé par le Père pour le salut du monde: Jonas jeté dans les flots, c'est le Christ livré à la mort bien qu'innocent: Jonas englouti puis rejeté par le poisson. c'est le Christ mort puis ressuscité le troisième jour ${ }^{116}$.

La plaque de l'autel de Stavelot c'est enfin l'ECCLESIA couronnée, tenant la croix et le calice. opposée à la SINAGOGA. les yeux bandés, tenant les instruments de la Passion. lance et bâton avec l'éponge dans sa main droite, couronne d'épines dans sa main gauche, dans les parties supérieure et inférieure du quadrilobe central. La première représentation de l'Église et de la Synagogue autour d'une Crucifixion est dans une série d'ivoires des $I X^{\mathrm{C}}-\mathrm{X}^{\mathrm{C}} \mathrm{s}$. de Metz ou de sa région pour des plats de reliure: la Synagogue n’est pas encore un personnage déchu. elle se détourne seulement de la Croix. À partir de la fin du $\mathrm{XI}^{\mathfrak{E}}$ s. la Synagogue va être représentée très négativement : la couronne tombe de sa tête, sa bannière est brisée, un bandeau couvre ses yeux. et parfois, comme à Stavelot, elle porte les instruments de la Passion et donc est signifiée comme portant la responsabilité de la mort du Christ ${ }^{117}$. Cette responsabilité est ici clairement affirmée par la présence, sous la Synagoga, de la comparution au Christ devant Pilate qui dit : INNOCENS EGO SUM A SANGUINE (Justi hujus), "Moi je suis innocent du sang de ce juste". alors que les juifs répondent : SANGUIS EJUS SUPER NOS ET SUPER FILIOS NOSTROS, "Que son sang [retombe] sur nous et sur nos enfants" (Mt XXVII, 24 et 25). Linscription qui court sur le rebord de la plaque supérieure de l'autel commente très précisément toute cette iconographie et son accompagnement épigraphique :

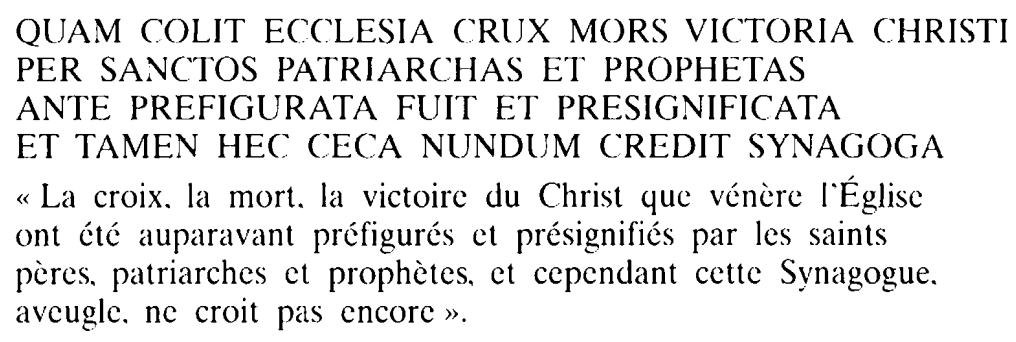

L'aveuglement de la Synagogue, maintes fois rappelé, est particulièrement souligné dans la Disputatio Ecclesiae et Synagoguae auctore Gillieberto. du XII ${ }^{2}$ s., publiée par Martène et Durand ${ }^{118}$. Dans le dialogue l'Église appelle la Synagogue sa "mère", mais la dit surda mater et caeca, mater surda et caeca, mater caeca et misera, sterilis et arida. Sur la châsse de saint Eleuthère à la cathédrale de Tournai la Synagogue est aussi dite "aveugle (CECA RUENS SYNAGOGA PERIT FRUSTATUR HONORE).

114. Jon II. 1 et l'ensemble des chapitres I et II.

115. Mt XII. 38-41. XVI. 4: Lc XI. 29-32. col. 125).

116. Par exemple. vers l'époque de lautel de Stavelot. Rupert de Deutz dans son De divinis officiis (PL, 170).

117. Bernhard Butmenkrav\%. "Synagoga méconnue. Synagoga inconnue". Revue des études juives. 125. 1966. p. 35-49: - ID.. Le juif médiéval an miroir de l'art chrétien. Paris. 1966. p. 105-108: - ID. "La représentation de la Sinagega dans les Bibles moralisées françaises des xulle et xve siècle ". Israel Academy of Sciences and Humanities Proceedings. V. 1970. p. 70-91.

118. Thesaturus novus anecdotorum. V. Paris. 1717. col. 1497-1506: sur un autel portatif de Darmstadt on avait aussi : ... CAFCA FUCIIT SYNAGOGA (J. BRAL [op) (it. n. 2]. 1. p. 475). 
Sur les côtés de laautel portatif sont représentés les douze apôtres avec l'indication de leur martyre : MARTIRIUM PAULI. CRUCIFIXIO PETRI. DECOLLATIO JACOBI. etc. Les passions des douze apôtres rassemblées par le pseudo-Abdias au VIC s. reprises au XIII dans la Légende dorée. ne sont pas souvent représentées dans l'iconographic. Le Christ avait annoncé aux apôtres quils "boiraient le calice qu il allait boire" (Mc X. 39). Par leur martyre ils ont témoigné de la Bonne Nouvelle que le Christ les avait chargés denseigner à toutes les nations. Linscription placée sur le bord inférieur de lautel portatif en quatre vers comme la précédente. concerne à la fois les apôtres et les quatre évangélistes représentés aux quatre pieds de l’autel :

\section{HI QUE SCRIPSERE DOCTORE DEO DIDICERE \\ HORUM FIRMATA PLAGIS ET MORTE PROBATA \\ ET CELEBRATA SIMUL HORUM DIVINITUS ORE ISTORUMQUE PIO PARITER SANCCITA CRUORE}

"Ce que ceux-ci ont écrit ils l'ont appris de l'enseignement de Dieu.

ils l'ont confirmé par leurs supplices et vérifié par leur mort.

et a été aussi bien célébré par leur bouche inspirée par Dieu

que consacré par le pieux sacrifice de ceux-là ".

Ce témoignage par le sang des apôtres est aussi confirmé par la sainteté de ceux qui ont suivi le message de l'Évangile, les saints dont les reliques ont été placées dans lautel. comme le dit sobrement l'inscription au centre de la plaque inférieure : RELIQUIE SANCTORUM. La châsse de saint Éleuthère à Tournai rappellera plus tard elle aussi que les apôtres ont été martyrs et évoquera les différents genres de martyre qu ils ont subis ${ }^{119}$.

L’autel portatif du Musée diocésain d’Augsbourg porte aussi la représentation de l'ECCLESIA et de la SYNAGOGA - yeux bandés, portant couronne et lance brisée - que l'on retrouve sur l'autel portatif de l'église St. Vitus de Mönchen-Gladbach ${ }^{120}$, au reste très proche d'esprit de l'autel St. Mauritius de Siegburg. Dans cette ouvre de Cologne, vers 1160, dix scènes se succèdent autour de la pierre d'autel en vert antique. En haut sont représentés l'Offrande de Melchisédech (Gn XIV, 18), le Sacrifice d'Isaac par Abraham (Gn XXII. 9), le Sacrifice d'Abel (Gn IV, 4). Ce sont les trois figures vétéro-testamentaires rappelées par le prêtre au canon de la messe après la consécration. Un distique léonin riche en donne le sens :

\section{MUNERA TERNORUM SIGNANT HEC TRINA VIRORUM}

OB NOS OBLATUM SUMMI PATRIS IN CRUCE NATUM

"Cette triple offrande des trois hommes sont le signe

du Fils du Père éternel offert pour nous sur la croix".

Peut-être y a-t-il. dans cette façon de souligner le chiffre trois, une intention dévoquer la Trinité pour laquelle. depuis Augustin. on emploie l'adjectif trini. Sur les côtés latéraux de la pierre dautel sont figurées quatre annonces vétéro-testamentaires de la Crucifixion. dont la messe fait mémoire. Il y a d'abord Mö̈se et le serpent d'airain (Nb XXI. 8-9). figure, rappelons-le, que Jésus lui-même a donnéc comme annonce de sa mort sur la croix (Jn III. 14). Mö̈se tient un phylactère sur lequel est écrit : OS NON COM[MINUETIS] EX EO. La référence première est dans les prescriptions concernant la Pâque que Yahvé donne à Mö̈se : hec os illius confringetis (Ex XII. 46) : "Vous ne romprez aucun os de la victime». c'est-à-dire de l'Agneau pascal évoqué au début de ce même chapitre. La prescription est renouvelée par Yahvé à Moïse : os éjus non confringent dans le livre des Nombres (IX. 12). Le Psaume XXXIV (XXXIII), 21 affirme que Yahvé protège les justes de tout malheur : "Yahvé garde tous leurs os. pas un ne sera brisé ". custodit Dominus omnia ossa eortum. unum ex his non conteretur. La citation de Mönchen-Gladbach vient en fait de l'Évangile de Jean : les soldats brisent les jambes des deux larrons mais non celles de Jésus car il était déjà mort. "Car. ajoute Jean. cela est arrivé pour que

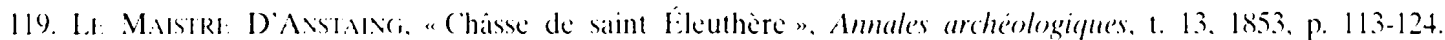

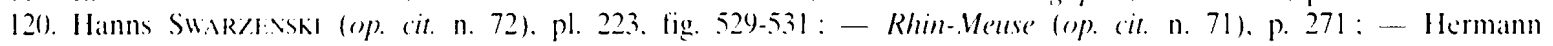
S(HNTHIFR (op) (it. n. 91). n 36. p. 4t.46. 
s’accomplisse l'Écriture : On ne lui brisera pas un os". os non comminuetis ex eo (XIX. 36). (e rapprochement conduit à faire du Christ la nouvelle victime de la Pâque par sa mort sur la croix. L’évangéliste ajoute : "ailleurs l'Écriture dit encore : Ils regarderont celui qu ils ont transpercé ". videbunt in quem transfixerunt (XIX. 37). Cette fois c'est une citation du prophète Zacharic (XII. 10)). Aspicient ad me quem confixerumt et sous Moïse et le serpent d'Airain est représenté Zacharie avec sa citation telle que la donne Jean. Du côté droit de la pierre d'autel ont été représentés Job et Isaïe. Job est la figure même de la patience (IV. 6: XVII, 15) et il tient en ses mains le bouclier de la PATIENTIA. Déjà Zénon et saint Jérôme donnent Job comme la "figure du Christ "121. et Grégoire le Grand comme Rupert de Deutz y voient un modèle de "la patience dans l’adversité "122. Quant à Isaïe. il dit, sous une forme abrégée SIC. $\mathrm{O}$. AD O., une annonce du Sacrifice, sicut ovis ad occisionem "comme la brebis est conduite à l’abattoir» (LIII. 7). que l’apôtre Philippe applique déjà au Christ lorsqu îl explique le passage à l'eunuque éthiopien (Actes. VII, 31). Dans son commentaire sur Isaïe. Jérôme verra aussi dans le Christ l'agneau conduit devant Pilate et qui se tait. et il sera repris par Prosper d'Aquitaine, Haymon d'Halberstadt. Rupert de Deutz. Hervé de Bourg-Dieu ${ }^{123}$. Le verset d'Isaïe sert de répons aux matines du samedi saint ${ }^{124}$.

On a donc suivi ici Grégoire le Grand et dautres auteurs qui conseillaient de prouver aux juifs la véracité de l’enseignement chrétien à l’aide de leurs propres livres ${ }^{125}$. El dès lors, dans les deux figures qui entourent la Crucifixion en bas de la plaque supérieure de l'autel de MönchenGladbach. il faut voir la victoire de l'Église à gauche - donc à la droite du Crucifié - couronnée. nimbée. avec calice et croix triomphale. sur la Synagogue. qui. les yeux bandés. tient lance et bâton avec l'éponge. instruments de la Passion d'une main. et Tables de la loi de l'autre. Le commentaire en hexamètres léonins est parfaitement clair à ce sujet :

GAUDEAT ECCLESIA DIRA DE MORTE REDEMTA

LEGIS SUMMA PERIT DUM MUNDUM VITA REDEMIT

"L'Église se réjouit d’être rachetée d’une mort cruelle.

la somme de la Loi périt lorsque la vie racheta le monde".

Sur les longs côtés sont représentés les douze apôtres, identifiés par leurs noms. Sur lun des petits côtés le Christ en majesté (MAJESTAS) est entouré de Marie et Jean Baptiste à sa droite. des saints Michel et Étienne à sa gauche. Sur lautre petit côté le sépulcre vide (SEPULCHRUM) sur lequel veille un ange est entouré des trois saintes femmes du matin de Pâques (MULIERES) et des soldats endormis (CUSTODES). On notera la particularité paléographique de la conjonction du V et du $S$ (IS) que l'on observe aussi dans un autel portatif de l'église Saint-Michel de Brunswick ${ }^{126}$ et dans le nom de Meinwercus sur l'autel portatif de Roger d'Helmarshausen au musée diocésain de Paderborn.

La présence fréquente des apôtres sur ces autels portatifs est peut-être due aussi au fait que ces autels sont destinés souvent à des évêques. successeurs des apôtres, et comme eux chargés d’annoncer la Bonne Nouvelle. ce pour quoi d’ailleurs généralement ils se déplacent et ont besoin d'autels portatifs. Sur un autel de Cologne du troisième quart du XII s. conservé au musée de Darmstadt ${ }^{127}$ les douze apôtres sont ainsi représentés - sauf Jean. Philippe et Simon - avec le pays qu ils ont évangélisé : Pierre Rome. Paul la Grèce. André l'Achaïe. Jacques (le Mineur) Jérusalem. Thomas l'Inde. Barthélemy l'India tertia. Jacques la Samarie. Matthieu l'Éthiopie. Jude l'Égypte. Différents auteurs ont donné des listes des pays de missions des apôtres. Fortunat.

121. Pl. 11. col. 11-41. Iractatus XV. de Joh. t. 28. col. 619-620) et ss. (ommentarii in librum Joh.

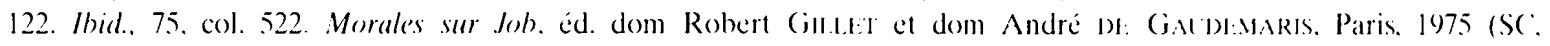
32 his): - PL. 167. col. 1607. exemplar patientiae. ou 168: Super Job commentarius.

123. C.I.I.M. t. 18. Paris. 1995. p. 124.

124. Corpus amtiphomaitum officii (op). cit. n. 78). n 7661. p. 409

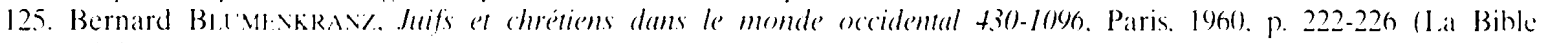
it son emploi)

126. Die Inschrifien der Stadt Braunschweig (op. cit n. 53). fig. 12.

127. ('f. supra n. 34 . 
Julien de Tolède. Isidore de Séville. Beatus de Liebana. et on peut y joindre le Breviarum gothicumm $^{128}$. Il y a un consensus général pour attribuer Rome à Pierre. l'Achaie à André. Jérusalem à Jacques le Mineur. l'Inde à Thomas - sauf Fortunat qui lui donne Édesse: I'Espagne est habituellement donnée à Jacques le Majeur: Julien de Tolède attribue l'Éthiopie à Matthieu. que généralement on voit en Macédoine: à Jude va la Mésopotamie plutôt que l'Égypte habitucllement associée à Simon. Le pays n'est pas ici donné pour Jean qu'on place à Êphèse ou plus globalement en Asie. ni à Philippe. évangélisateur de la Galatie.

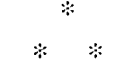

Le XII siècle est ainsi marqué par un renouveau du prophétisme. de plus fortes références à l'Ancien Testament ${ }^{129}$. même si ces références se rencontrent avant cette date, ainsi d'un autel portatif clu musée national du Moyen Âge à Paris, du début du XI' s. qui met en scène le sacrifice d'Abraham (ABRHAM). et les deux figures de prêtres. MELCHISEDECH et AARON ${ }^{1,30}$. Ce renouveau du prophétisme et de la mise en concordance de l'Ancien et du Nouveau Testament. a. pour une part. une explication dans le contexte de controverses judéo-chrétiennes particulièrement fréquentes de la fin du $\mathrm{XI}^{\circ}$ au début du XIII' $\mathrm{s}$. Un autre trait des inscriptions des autels portatifs de la fin du XI' et du XII' $\mathrm{s}$. est la conséquence des débats qui eurent lieu à la suite de l'enseignement de Bérenger de Tours sur l'eucharistic. Le savant écolâtre, en effet, rejetait la présence réelle du corps et du sang du Christ dans les espèces consacrées du pain et du vin. ce qui avait entraîné une série de condamnations conciliaires et qui provoquera un évident approfondissement théologique du sacrement de l'eucharistie à cette époque. On en trouve l'écho dans les inscriptions des autels portatifs. comme d’ailleurs des calices et patènes. de cette époque. Un des plus importants autels portatifs du XII" S., celui dit de Saint-Grégoire au trésor de S. Servatius de Siegburg. en offre un bon exemple ${ }^{1.31}$.

Lautel de Saint-Grégoire porte, sur les côtés, dix-huit prophètes, avec leurs noms sur des phylactères. Autour de la pierre dautel en serpentine huit scènes anépigraphes illustrent la vie du Christ (Annonciation. Nativité. Adoration des mages. Présentation au temple. d'un côté. Baptême de Jésus. Lavement des pieds à la dernière Cène. Crucifixion. les Saintes femmes au tombeau de lautre). Tout autour de la plaque supérieure sont les figures de trente-deux saints et saintes :

- le long dun grand côté les apôtres : Mathias, Juclas, Matheus, Andreas, Jacobus, Petrus, Johannes, Simon. Thadeus, Bartholomeus. Tomas, Paulus:

- Ie long de lautre grand cóté douze évéques. avec en tête cinq saints évêques de Cologne du ve au XI" s. : Cumibertus, Heribertus, Brumo, Severinus, Evergislus, Martimus, Ambrosius, Bricius, Dunstanus, Nicolaus, Servatius, Augustinus:

- Ie long d'un petit côté quatre martyrs "militaires": Mauricius, Geréon. Géorgius, Mercurius:

- Ie long de lautre petit côté. quatre vierges martyres : Cecilia. Ursula. Agatha. C'aterina.

Deux inscriptions. de quatre vers commentent le sacrifice célébré sur l'autel. Autour de la pierre d'autel et commençant au milieu d'un long côté - entre les apôtres Picrre et Jean - on lit :

\author{
+ ARA CRUCIS CRISTI MENSE COMMUNICAT ISTI \\ HAC ETENIM RITE SACRATUR VICTIMA VITE \\ IN QUA STRUCTLRA VIRTYTUM NON RUITL;RA \\ PONITUR HAC DOMINO DINGNA DOMUS STRUITUR.
}

128. PI. S8. col. 270 (Fortunat): 96. col. 746 (Julien): 81 . col. 389-390) (Isidore) et 392-393 (Beatus): 86 . col. 13061307 (Bretarium gothicum au 30 décémbre).

129. Aux autels déja cités on peut joindre un autel portatil du musée diocésain d'Augshourg. du miliéu du vil" s. ou. aux quatre angles. sont représentées quatre figures vétéro-testamentaires de la (rucifixion et du Sacrifice de la Messe.

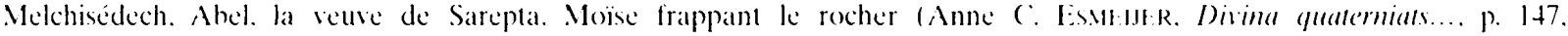
n. $1+5$.$) .$

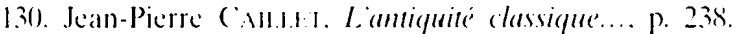

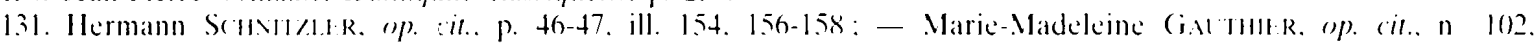

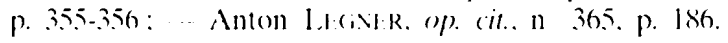


« L Lautel de la croix du Christ est en relation avec cette table:

par lui en effet à juste titre est consacrée la victime de vie.

en qui repose toutes les vertus et qui ne périra pas.

Par elle une digne maison est construite pour le Seigneur».

Trois vers léonins riches et un hexamètre simple mettent ainsi en relation. en une formulation compliquée. le sacrifice du Christ sur la croix et sa commémoration dans l'eucharistie. L'expression ara crucis est aussi employée dans une hymne à la croix ${ }^{1.32}$. On trouve les mêmes deux premiers vers inscrits sur la plaque supérieure d’un autel portatif (v. 1180)-1200) au trésor de la cathédrale de Bamberg ${ }^{133}$. Sur le bord extérieur de la plaque quatre autres vers ont été gravés :
$\dagger$ QUICQUID IN ALTARI TRACTATUR MATERIALI
CORDIS IN ALTARI CONPLETUR SPIRITUALI
HOSTIA VISIBILIS MACTATUR OPERTA FIGURA
INMOLAT HANC PURA DEVOTIO MENTIS IN ARA
" $\dagger$ Ce qui est traité sur l'autel matériel
trouve son accomplissement en l'autel spirituel du ceur.
Une victime visible est sacrifiée sous une forme voiléc.
Une pure disposition de l'esprit l'immole sur l'autel".

I.e premier distique est en vers léonins riches. le troisième vers est un simple hexamètre. le dernier est un vers léonin. Ces quatre vers ont aussi été inscrits sur un reliquaire émaillé de SaintVictor de Xanten, de la fin du XII ${ }^{\text {s. }}{ }^{134}$. On a, là encore sous une forme savante, voulu souligner qu'il y avait un nécessaire mouvement de foi pour passer des apparences matérielles à la signification profonde de la consécration cucharistique. C"est un type d'inscription qu'ignoraient les autels portatifs antérieurs à la polémique bérengarienne. Le décret de Gratien insistera sur la signification "spirituelle ". c'est-à-dire sur le mouvement de foi qui conduit à voir, après la consécration, sous la réalité "matérielle" du pain et du vin. le corps et le sang du Christ : Non carnaliter set spiritualiter Christi corpus et sanguinem debemus accipere, spiritualiter magis quam corporaliter corpus Christi debenus accipere, in sacrificio non corporalem sed spiritualem escam accipimus ${ }^{135}$.

Sur le bord de la plaque supéricure d’un autel portatif de Sainte-Marie-au-Capitole de Cologne ${ }^{1.36}$. on a gravé un distique très proche de celui inscrit sur l'autel de Saint-Grégoire de Siegburg.

\section{QUICQUID IN ALTARI PUNCTATUR SPIRITUALI \\ ILLUD IN ALTARI COMPLETUR MATERIALI \\ "ce qui est signifié sur l'autel spirituel \\ est accompli sur lautel matériel».}

Les angles sont ensuite occupés par les figures du tétramorphe. JOHANNES ct MATHEUS en haut. MARCUS et LUCAS en bas. Un second distique entoure la pierre dautel centrale en serpentine qui. de surcroît. est encadré des figures d'Abel offrant l'Agneau et de Melchisédech tenant le vin et le pain.

\section{ARA CRUCIS TUMULI [QUE] CALIX LAPIDISQUE PATENA SINDONIS OFFICIUM CANDIDUS BISSUS HABET}

"Lautel tient licu de la croix. le calice du tombeau.

la patène de la pierre. Ie lin blanc du suaire".

132. Analecta hymnica Medii Aevi, t. 8. éd. (juy Marie DrI:vEs. lepizig. 1890, n 21. p. 26.

133. Adolf (jolioschinit) (op. cit. n. 30). n 28, p. 28. pl. XXX et XXXI.

134. Die christlichen Inschriften der Rheinlande von der mitte des achten bis zur mitte des dreizehmen Jahrhunderts. éd. Fran\% Xaver Kral's. Fribourg-en-Br./Leipzig. 1894, n 656. p. 301.

135. Corpus juris camonici... l. ed. E.L. Richter et E. FrIledber(j. Leiprig. 1922. c. 1330) 1338. 1349.

136. Die (hristlichen Inschriften... (op. cit. n. 134), n 572, p. $268:-$ Rhin-Meuse'.. (op. (it. n. 71 ) p. $274:-$ Jospeh BralN (op. (it. n. 2). I. p. 481-482. Lautel est déposé au Schnütgen Museum. 
Il saagit d'un distique élégiaque. donc d'une composition métrique franchement différente du premier distique en hexamètres léonins riches. On a donc emprunté à deux sources différentes. la dernière venant d'un poète unanimement reconnu au XII' s. Hildebert de Lavardin. dans son $D e$ expositione missae ${ }^{1.37}$, traité liturgique largement diffusé au Moyen Âge ${ }^{138}$. Hugues de Saint-Victor. son contemporain. a exprimé les mêmes analogies dans son De sacramentis fidei christianae : Ara crucem significat, calix sepulcrum, patena lapidem, corporalis palla sindonem qua involutum est corpus Christi ${ }^{1.39}$. mais c'est la formule plus ramassée. d'Hildebert. exprimée en vers donc plus aisée à mémoriser. qui a eu le plus de succès. Le chanoine régulier sous-prieur de Sainte-Barbeen-Auge. Geoffroi. cite les deux vers de l'egregias versificator. dans une de ses lettres ${ }^{1+1}$. et lévêque de Crémone. Sicard. donne aussi le distique dans la brevis recapitulatio missae du livre III du Mitrale ${ }^{1+1}$ Pierre le Peintre. chanoine de Saint-Omer autour de 1100) avait déjà approché cette explication symbolique dans son Liber de sacra eucharistia :

Ara crucis lignum. signatque calix monumentum.

un livre que les éditeurs de la Patrologie latine ont publié à la fois dans les ouvres d'Hildebert de Lavardin et de Pierre de Blois ${ }^{142}$. La référence au lin comme image du suaire renvoie aux multiples prescriptions données dans le livre de l'Exode pour le sanctuaire (demeure. voile. rideaux du parvis) et les vêtements des prêtres ${ }^{143}$. prescription reprise pour la célébration eucharistique par le décret de Gratien ${ }^{144}$. Les deux vers d'Hildebert ont été aussi inscrits sur un autel portatif. disparu. de La Souterraine en Limousin au XII s. el sur la patène trouvée dans la tombe de larchevêque de Cantorbéry Hubert Walter. mort en $1205^{14.5}$.

Sur un autel portatif du musée de Berlin, de la fin du XI' s. une inscription dit clairement que la main du prêtre est l'intermédiaire par lequel "le pain devient hostie de vie " ${ }^{1 \text { th }}$ et. du début du XIIC s.. l'autel portatif de Roger d'Helmarshausen au musée diocésain de Paderborn ${ }^{147}$. présente l'évêque saint Meinwerk dans son office de célébrant au moment de la communion : CALICEM SALUTARIS ACCIPIAM ET NOMEN DOMINI INVOCABO. "Je prendrai le calice du salut et j'invoquerai le nom du Seigneur ». Ce texte est dit par le prêtre avant de communier au sang du Christ: c'est une citation du Psaume CXVI, verset 1.3 (Vulgate CXV, 3), auquel renvoient les récits de la dernière Cène dans les Synoptiques : le Christ prend la coupe (calice accepto) et rend grâces. Comme dans l'autel de Paderborn la référence à l'eucharistie et à la présence réelle n'est qu'une mention parmi de nombreuses inscriptions. ici consacrées aux saints vénérés dans l’autel. sur l'autel portatif d’environ 1140/50) provenant d'Hildesheim, conservé au Victoria and Albert Museum à Londres ${ }^{148}$. Autour de la plaque de porphyre sont représentées des scènes de la vie du Christ. de sa naissance à sa résurrection. et douze bustes de saints qui sordonnent autour du buste du Christ au milieu du long côté supérieur et du buste de

137. Pl. . 171. col. 1194.

138. Dictionnaire des lettres françuises. l.e moyen äge. réd. Geneviève Hastonohr et Michel Zask. Paris. 196t. p. 681-682: louvrage de Hans Watste. Carmina medii aevi postrioris latina. I : Initia carminum ac lersumm medii aevi posterioris latinorum.... (iöttingen. 1959.

139. Pl. 176. col. 438 .

140. Ihid.. 205. col. 866. (pistola XXXIV ad R. presbiterum ret col. 865-866. egregius versificator).

1+1. Mhid.. 213. col. 1+6.

142. Ihid.. 171. col. 1212 (Hildebert): 207. col. 11.54 (Pierre de Blois). Voir E. ANAM. "Pierre le Peintre". dans IDictionnuire de theologie catholique. XII-2. Paris. 1935. col. 2036-20)37.

14.3. I:x. 25-28. 35-39. Jérôme (P1. 25. col. 87-89). Raban Maur (ibid.. 110. col. 6.30). Rupere de De:uty (ihid. 168. col. 1.368) commentent les vêtements de lin du grand prêtre.

14t. Corpus juris canonici. 1. col. 1.306.

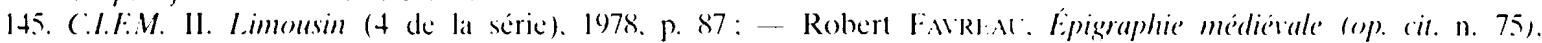
p. $153-155$

146. $\div$ DEXTRA SACERDOTIS (OMMENDAT MIONERA VOTIS

Q QIOD DATLR HO( LAIPI)E DH: PANE HIT HOSIIA VITH:

(I)ie Inschrifken det Studt Braunschweig bis 1528 (op. (it. n. 28). n 8. p. 11-12. fig. 8). [.autel est conserve au Kunstgewerbemuscum de Berlin.

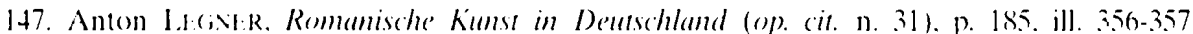

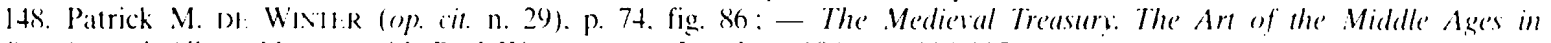

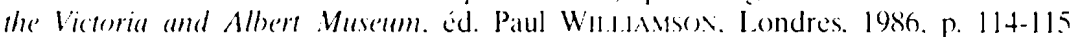


Marie (MATER MISERICORDIE) sur l'autre long côté. Le texte qui accompagne le Christ est une phrase dans laquelle il annonce la dernière Cène et fonde la foi chrétienne en la présence réclle dans l'eucharistie : PANIS QUEM EGO DABO CARO MEA EST. "Le pain que je donnerai est ma chair (pour la vie du monde) (Jn VI. 52). Dans son De sacro altaris mysterio. Innocent 1II. posant la question :" Est-ce que la substance du pain devient le Christ?" (Utrum panis transsubstantietur in Christum?) écrit ${ }^{149}$ : "Je suis, dit-il, le pain vivant descendu du ciel. et le pain que je donnerai est ma chair pour la vie du monde (Jn VI). Le Christ, en effet, appelle sa propre chair du nom de pain. afin de montrer par là que le pain est comme vraiment changé en sa chair. et que le pain devient lui-même" (Christus igitur seipsum et carnem suam nomine panis appellat, ut ostendat ex hoc quod panis sicut vere mutatur in carnem ipsius, ita vere mutatur in ipsum).

Un autel portatif conservé jusqu'en 1790 à la cathédrale d'Oloron-Sainte-Marie ${ }^{150}$. ouvre du temps de lévêque Roger (1101-1114), porte des inscriptions en relation directe avec la présence réelle dans l'cucharistie. mise en doute par Bérenger de Tours. Sur la face supérieure on pouvait lire:

\section{RES SUPER IMPOSITAS COMMUTAT SPIRITUS ALMUS \\ FIT DE PANE CARO SANGUIS SUBSTANTIA VINI \\ SUMTA VALENT ANIMAE PRO CORPORIS ATQUE SALUTE \\ "Le Saint Esprit transforme les offrandes ci-dessus placées : \\ le pain devient chair. la substance du vin devient sang. \\ Ils procurent le salut de lâme et du corps à ceux qui les reçoivent ».}

Sur la face inféricure on avait ajouté :

\section{DANTUR IN HAC MENSA SANGUIS CARO POTUS ET ESCA VERBA REFERT CAFNAE SUPER HAEC OBLATA SACERDOS MUNERA SANCTIFICAVIT ET PASSIO COMMEMORATUR}

"Sur cette table sont donnés le sang et la chair, breuvage et nourriture.

Le prêtre renouvelle les paroles de la (ène sur ces offrandes.

Il sanctifie ces présents. et la Passion est commémorée».

Ici ce qu'on appellera au XII" s. la "transsubstantiation" est clairement affirmée. à l'encontre de la position de Bérenger : le pain devient chair du Christ. le vin son sang. caro enim mea vere est cibus et sanguis meus vere est potus, comme l'a affirmé le Christ (Jn VI, 56). Paul parlait déjà de "nourriture spirituelle". escam spiritalem (1 Cor X. 3) pour la manne, figure de l'eucharistic. et saint Ambroise reprendra le mot, esca caelestis ${ }^{151}$. Le verbe sumere renvoie au récit de la Cène par Marc : Jésus "prit du pain... et le leur donna en disant : Prenez. ceci est mon corps". sumite : oac est corpus meum (XIV, 22). La transformation par l'Esprit Saint. la médiation du prêtre. laffirmation de la présence réelle, citées dans les inscriptions de cet autel renvoient parfaitement à la confession du concile de Poitiers de 1075 au cours des controverses avec Bérenger : Credimus corde et ore confitemur panem illum et vinum illut que in altari ad sacrificandum ponuntur, ipsum eundem panem et ipsum idem vinum, post consecrationem que ibi fit virtute Spiritus Sancti per manum sacerdotis, substantialiter transmutatum in verum corpus et in verum sanguinem Christi ${ }^{152}$. Le sacrement de l'eucharistie est "commémoration de la Passion du Christ ", souligne le Décret de Gratien 153. "commémoration de la mort du Christ ". reprendra Innocent III ${ }^{154}$. ("est en fait un rappel du commandement du Seigneur lors de la Cène. selon la première mention qui en est faite. à savoir celle de Paul dans la première épître aux Corinthiens (XI. 24 et 25) : hoc facite in meam commemorationem.

149. PL. 217. col. 869-870.

150. (IIF. 6. 6. p. 161-162. Certains auteurs nont pas voulu y voir un autel portatif. Les inscriptions semblent bien leur donner tort (res saper impositas. in hac mensa).

151. Pl. 16. col. $9(6)$.

152. Robert Sont:RVII...1. "The (ase against Berenger of Tours. A New Tex1". Studl (jregoriani. IX. 1972. p. 68,

15.3. Corpus juris canomici. 1. col. 1.341.

1.54. Pl. 217. col. sk? 
À la suite de linscription de consécration de 1187 on lisait. sur un autel portatif de Saint-Laurent de Liège ${ }^{155}$ un distique léonin riche :

HIC DATUR IPSE JESUS ANIMARUM POTUS ET ESUS

HAEC TIBI SIT CARA CUI CARO FIT CRUCIS ARA

"Ici Jésus lui-même est donné en breuvage et nourriture des âmes.

Que cet autel de la croix te soit cher. à toi pour qui Jésus devient chair».

Le mot esus est employé pour l'agneau que doivent manger les Hébreux avant leur départ d’Égypte sous la conduite de Mö̈se (Ex XII. 4). mais a sans doute été ici retenu plutôt pour des raisons de rime. On a, pour des raisons de métrique. aussi inversé l'expression ara crucis déjà rencontrée. Le jeu de mots caro cara est un exercice que l'on aime bien à l'époque de la vogue du vers léonin. Le message du texte est en tout cas le même que celui de l'autel d'Oloron : après la consécration des espèces du pain et du vin. c'est le corps et le sang du Christ qui sont sur lautel. nourriture et breuvage pour les âmes. Dans une jolie formule Baudri de Bourgueil termine une inscription pour un autel portatif par :

Christus edet tecum, si Christum vivis edendo.

"Le Christ mangera à ta table. si tu te nourris du Christ "15n.

Dautres inscriptions d'autels portatifs peuvent complëter ce dossier. C"est vraiment le corps du Christ qui est reçu dans l'eucharistic :

\section{IHESU CHRISTE TUI MISTERIA CORPORIS ALMI \\ DA SUMENDA NOBIS ET CLEMENS SACRA CRUORIS}

"Jésus (hrist. en ta clémence. donne-nous de recevoir

les mystères sacrés de ton saint corps et de ton sang"

(autel portatif de Melk. seconde moitic du XIe s.) ${ }^{157}$

$\dagger$ HOC DOMINI SACRA CORPUS MACTATUR IN ARA

QUO SUMPTO VIVIT DIGNUS, REUS INDE. PERIBIT

« $\dagger$ le corps du Seigneur est sacrifié sur laautel sacré.

En le recevant celui qui en est digne a la vie. celui qui en est indigne aura la mort».

(autel portatif de la cathédrale de Modène. XIIe s.) (.5. $^{15}$

référence à laavertissement de Paul aux Corinthiens (1 Cor XI. 27 : quiconque mange le pain ou boit la coupe du Seigneur indignement aura à répondre - reus erit du corps du sang du Seigncur).

"le pain devient hostic de vie" : DE PANE FIT HOSTIA VITAE

(autel portatif du Kunstgewerbemuseum de Berlin. XIIe s.) ${ }^{159}$.

Le saint Sacrifice est nourriture et breuvage - POTUMQUE CIBUMQUE - (autel portatif de la cathédrale de Spire. fin $X_{I I^{*}} \mathrm{~s} .{ }^{1(1)}$. et il doit être compris de façon systématique - HSO'IIA QUAE RITE SIGNATUR MYSTICA PER TE (autel portatif d'Eichstätt) ${ }^{161}$. Il faudrait ici trajter, avec les inscriptions des autels portatifs. des inscriptions des calices et des patènes.

Ainsi le message épigraphique des autels portatifs de la fin du XI" et du XIÍ s. porte-t-il deux traits caractéristiques : le recours aux prophètes et aux personnages vétéro-testamentaires, pour

155. Vosage litteraire de deux beineidictins. (op). cit. n. 4(3). p. 190. p. $1+1$.

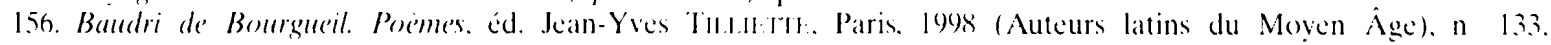

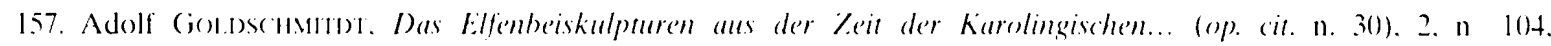
pl. XXXIII

158. Joseph Bral $x$ (op. (it. n. 2). I. p. 164.

159. Die Inschriften der Stadt Bratuschueig... (op) . (it. n. 28). n 8. p. 11-12.

160). Jospeh Brill $\times(o p$. (it. n. 2). 1. p. 315 .

161. Ihid.. p. $46+-465$. pl. 97. 
une part sans doute lié aux controverses judéo-chrétiennes de ce temps, et une affirmation plus théologique du sacrement de l'eucharistie. cette fois sûrement liée aux positions aventureuses de Bérenger de Tours sans cesse rappclées au XII" s. Il reste qu'on peut aussi trouver pour cette époque des inscriptions qui sont à mettre en relation avec la célébration de la messe. comme sur l'autel portatif de Melk, de la seconde moitié du XII' s. conservé à Washington. le rappel de "lagneau de Dieu qui enlève les péchés du monde "162. ou encore qui évoquent un passage de l'Évangile comme sur un autel portatif d'environ 1165. dont les quatre plaques émaillées des côtés seules conservées sont consacrées à la parabole des vignerons homicides (Mt XXI. 33-42) ${ }^{163}$. dans laquelle le fils du propriétaire de la vigne tué par les vignerons est évidemment une figure du Christ et de sa mort. On trouvera aussi bien des inscriptions qui intéressent le culte des saints. car les saints et leurs reliques sont très liées aux autels (portatifs ou non). Comme le dira un autel portatif d'Allemagne du Sud des environs de 1240) où vingt saints et saintes sont représentés sur les côtés ${ }^{164}$ : OMNES ISTI SANCTI QUI SUNT HIC INSCRIPTI ILLORUM SANCTUARIUM EST HIC. «Tous ces saints qui sont inscrits. leur sanctuaire est ici ». Mais avec le XIIl s. s’arrête la grande série des autels portatifs.

Robert FAVREAU:

Centre d’Études supérieures de Civilisation médiévale 24, rue de la Chaîne

BP 6() 3

F-86022 POTTIERS

162. Washington. Dumbarton Oaks collection :

* PIIS VALUIT ('UNCTIS JOHANNES VOCE PRECONIS

+ INQLIT EN AGNE DEI TOLLIT QUI CRIMINA MUNDI

" + Jean a valu plus que tous les autres, lui qui par sa voix de heraut

† annonce : Voici l'Agneau de Dieu qui enlève les péchés du monde".

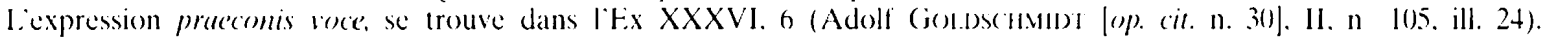

16.3. Ant mosan des $x r^{\prime}$ at $x / r^{\prime}$ siecles. Bruxelles. 1961. n 33. p. 210 : au musée du Bargello a Florence.

164. Munich. Schatzkammer der Residen\%. H. THIOMA (op). (it. n 12). p. $26-27$. 